ALEA, Lat. Am. J. Probab. Math. Stat. 14, 691-718 (2017)

DOI: 10.30757/ALEA.v14-33

\title{
A Mecke-type formula and Markov properties for STIT tessellation processes
}

\author{
W. Nagel, N. L. Nguyen, C. Thäle and V. Weiß \\ Institut für Mathematik, Friedrich-Schiller-Universität Jena, \\ Ernst-Abbe-Platz 2, \\ 07743 Jena, Germany. \\ E-mail address: werner.nagel@uni-jena.de \\ Via Giulio Tanini 6/6A, \\ 16133 Genova, Italia. \\ E-mail address: ngoclinhnguyen412@gmail.com \\ Fakultät für Mathematik, Ruhr-Universität Bochum, \\ Universitätsstr. 150, \\ 44780 Bochum, Germany. \\ E-mail address: christoph.thaele@ruhr-uni-bochum.de \\ FB Grundlagenwissenschaften, Ernst-Abbe-Hochschule Jena, \\ Carl-Zeiss-Promenade 2, \\ 07745 Jena, Germany. \\ E-mail address: viola.weiss@eah-jena.de
}

\begin{abstract}
An analogue of the classical Mecke formula for Poisson point processes is proved for the class of space-time STIT tessellation processes. From this key identity the Markov property of a class of associated random processes is derived. This in turn is used to determine the distribution of the number of internal vertices of the typical maximal tessellation segment.
\end{abstract}

\section{Introduction}

The last decades in stochastic geometry have seen a growing interest in models that deal with random geometric objects evolving in time. As examples we mention random sequential packings, see Penrose and Yukich (2002); Schreiber et al. (2007), spatial birth and growth models like the Johnson-Mehl growth process, see Baryshnikov and Yukich (2005); Penrose and Yukich (2002), the construction of polygonal Markov random fields, see Schreiber (2005, 2008, 2010), falling/dead leaf models, see Cowan and Tsang (1994); Bordenave et al. (2006); Galerne and Gousseau (2012),

Received by the editors March 8th, 2017; accepted July 25th, 2017.

2010 Mathematics Subject Classification. 60D05, 60G55, 60J75.

Key words and phrases. Markov Property, Maximal Polytopes, Maximal Segments, Mecke Formula, Poisson Point Process, Random Tessellation, STIT Tessellation, Stochastic Geometry. 
on-line geometric random graphs such as the on-line nearest neighbour graph in Penrose and Wade (2008); Wade (2009) or the geometric preferential attachment graph, see Jacob and Mörters (2015); Jordan (2010); Jordan and Wade (2015). A particularly attractive class of models studied in stochastic geometry is that of random tessellations. Also within this class, space-time models have found considerable interest. In the present paper we investigate the class of STIT tessellations, which arise as outcomes of a process of consecutive cell divisions. They have been invented in Nagel and Weiß (2005) and since their introduction they have stimulated lots of research, cf. Deuß and Thäle (2016); Lachièze-Rey (2011); Martínez (2014); Martínez and Nagel (2012, 2015, 2016); Mecke et al. (2011); Nagel and Biehler (2015); Nagel and Weiß (2008); Schreiber and Thäle (2010, 2011, 2012, 2013a,b); Thäle and Weiß (2010, 2013). The acronym STIT stands for the stability of distribution under the operation of iteration (or nesting) of tessellations.

The STIT tessellation process itself is a Markov process on the space of tessellations. However, there are several interesting situations in which the problem arises whether or not some classes of associated processes also possess the Markov property. For example, we look at the random process induced by the functional of total surface area within a bounded window. It becomes clear that this process does not inherit the Markov property from the STIT tessellation process, because this functional does not contain enough information about the tessellation. For this reason, it is an interesting task and one of the main purposes of this paper to extract a class of processes that do inherit the Markov property. Such a Markov property will turn out to be a crucial device in further applications. In the present paper we will deal with the distribution of the number of internal vertices of a typical (and possibly weighted) maximal segment of a STIT tessellation. In particular, we will derive the exact distribution of this random variable and study its moment properties.

One of the crucial steps on our way is to prove a Mecke-type formula for STIT tessellations. Such an identity is well known for Poisson point processes $\Gamma$. It says that the expectation of random variables of the form $\sum_{x \in \Gamma} f(x, \Gamma)$ can be expressed as an expectation of the integral with respect to the intensity measure of $\Gamma$ of the function $f\left(x, \Gamma+\delta_{x}\right)$, where the unit-mass Dirac measure $\delta_{x}$ concentrated at $x$ has been added to $\Gamma$, see Chapter 4 in Last and Penrose $(2017+)$ and also (3.2) below. Our key result, Theorem 3.1, provides a formula for STIT tessellations that is similar in its structure to Mecke's equation for Poisson point processes. We emphasize that our proof uses the global construction of STIT tessellations, which in turn is based on a Poisson point process on an abstract state space. As a consequence, our Mecke-type formula is based on the classical Mecke formula for Poisson point processes.

The present paper is structured as follows. In Section 2 we set up the notation, formally introduce STIT tessellation processes by their Markovian description and collect those properties that are needed. The new results are presented in Section 3. In particular, we present there our Mecke-type formula, the Markov properties described above as well as an application to maximal segments. The final Section 4 contains all the proofs. They are technically quite involved and they crucially depend on the 'direct' global construction of STIT tessellations. For this reason, we have also included a formal description of this construction together with its key properties. Our proofs show a high degree of formalism and technical computations. 
However, this seems unavoidable and is similar to other models studied in stochastic geometry, see, for example, the works Mecke and Muche (1995); Muche $(1996,1998)$ on Poisson-Voronoi tessellations.

\section{Preliminaries}

2.1. Notation. Let $\mathbb{R}^{d}$ be the Euclidean space of dimension $d \in\{1,2, \ldots\}$. A polytope $p \subset \mathbb{R}^{d}$ is the convex hull of a finite point set (containing at least two elements) and the dimension of $p$ is defined as the dimension of its affine hull. The set of all polytopes of dimension $k$ is denoted by $\mathscr{P}_{k}$. Moreover, we shall write $\mathscr{P}_{k}^{0}$ for the set of all $k$-dimensional polytopes with their circumcenter at the origin. The spaces $\mathscr{P}_{k}$ and $\mathscr{P}_{k}^{0}$ are supplied with the Borel $\sigma$-fields $\mathfrak{B}\left(\mathscr{P}_{k}\right)$ and $\mathfrak{B}\left(\mathscr{P}_{k}^{0}\right)$ induced by the Hausdorff-distance, respectively. In our context it is convenient to speak of the elements of $\mathscr{P}_{d}$ as cells and to denote them by the letter $z$ (for the German word 'Zelle'). The interior and the boundary of a set $B \subset \mathbb{R}^{d}$ are denoted by $\stackrel{\circ}{B}$ and $\partial B$, respectively. Moreover, we write \#(·) for the cardinality of the argument set and $\mathbf{1}\{\ldots\}$ for an indicator function, which takes the value 1 if the condition in brackets is satisfied and 0 otherwise.

The Lebesgue measure on $\mathbb{R}^{d}$ is denoted by $\ell_{d}$. Moreover, for $d=1$, we write $\ell_{+}$and $\ell_{-}$for the Lebesgue measure on the positive and the negative real half-axes $(0, \infty)$ and $(-\infty, 0)$, respectively. To simplify the notation in integrals, we write $\mathrm{d} s$ instead of $\ell_{+}(\mathrm{d} s)$.

If $\mathcal{E}$ is a topological space, we denote by $\mathfrak{B}(\mathcal{E})$ the Borel $\sigma$-field on $\mathcal{E}$. If $X$ is a random element taking values in the measurable space $[\mathcal{E}, \mathfrak{B}(\mathcal{E})]$ we shall write $\mathbb{P}_{X}$ for its distribution, that is, the image of the probability measure of some underlying probability space under $X$. By $X \stackrel{D}{=} Y$ we shall indicate that the $\mathcal{E}$-valued random elements $X$ and $Y$ have the same distribution, that is $\mathbb{P}_{X}=\mathbb{P}_{Y}$.

2.2. The hyperplane measure $\Lambda$. Let $\mathcal{H}$ denote the space of all hyperplanes in $\mathbb{R}^{d}$ and $\mathcal{H}_{0}$ be the subset of hyperplanes containing the origin. Both spaces are supplied with the usual topology of closed convergence (also called Fell topology, see Kallenberg, 2002, Chapter A.2, Last and Penrose, 2017+, Chapter A.3, or Schneider and Weil, 2008) and thus they carry Borel $\sigma$-fields $\mathfrak{B}\left(\mathcal{H}_{0}\right)$ and $\mathfrak{B}(\mathcal{H})$, respectively.

For $h \in \mathcal{H}$ we shall write $h_{0} \in \mathcal{H}_{0}$ for the parallel linear subspace and $h_{0}^{\perp}$ for the one-dimensional subspace orthogonal to it. The two closed half-spaces generated by a hyperplane $h \in \mathcal{H} \backslash \mathcal{H}_{0}$ are denoted by $h^{+}$and $h^{-}$, respectively, where we use the convention that $h^{-}$is the half-space that contains the origin. For a Borel set $B \subset \mathbb{R}^{d}$ we define

$$
[B]:=\{h \in \mathcal{H}: h \cap B \neq \emptyset\} \text {. }
$$

This implies $[B] \in \mathfrak{B}(\mathcal{H})$ and that $\mathcal{H}_{0}=[\{0\}]$.

Let $\mathbb{Q}$ be a probability measure on $\mathcal{H}_{0}$ and $\ell_{h^{\perp}}$ the Lebesgue measure on the subspace $h_{0}^{\perp}$. The translation invariant measure $\Lambda$ is defined by the relation

$$
\int_{\mathcal{H}} g(h) \Lambda(\mathrm{d} h)=\int_{\mathcal{H}_{0}} \int_{h_{0}^{\perp}} g\left(h_{0}+z\right) \ell_{h_{0}^{\perp}}(\mathrm{d} z) \mathbb{Q}\left(\mathrm{d} h_{0}\right)
$$

for all non-negative measurable functions $g: \mathcal{H} \rightarrow \mathbb{R}$. Throughout this paper we will assume that $\Lambda$ is such that there is no line in $\mathbb{R}^{d}$ with the property that all 
the hyperplanes in the support of $\Lambda$ are parallel to it. This ensures that, with probability one, all cells of the STIT tessellations considered below are bounded.

Because $\Lambda([q]) \in(0, \infty)$ for all polytopes $q \in \mathscr{P}_{k}$ with $k \in\{1, \ldots, d\}$, we can define the probability measure $\Lambda_{q}$ on $[\mathcal{H}, \mathfrak{B}(\mathcal{H})]$ by

$$
\Lambda_{q}(B)=\frac{\Lambda(B \cap[q])}{\Lambda([q])}, \quad B \in \mathfrak{B}(\mathcal{H}) .
$$

2.3. Tessellations. By definition, a tessellation $y$ of $\mathbb{R}^{d}$ is a countable subset of $\mathscr{P}_{d}$ satisfying the following three properties:

(i) $\stackrel{\circ}{z}_{1} \cap \stackrel{\circ}{z_{2}}=\emptyset$ for all $z_{1}, z_{2} \in y, z_{1} \neq z_{2}$,

(ii) $\bigcup_{z \in y} z=\mathbb{R}^{d}$,

(iii) $\#\{z \in y: z \cap C \neq \emptyset\}<\infty$ for all compact $C \subset \mathbb{R}^{d}$.

A 'local' tessellation $y$ of a polytope $W \in \mathscr{P}_{d}$ is a finite collection of polytopes contained in $W$ that have disjoint interiors and cover $W$. The set of tessellations of $\mathbb{R}^{d}$ is denoted by $\mathcal{T}$ and we write $\mathcal{T}_{W}$ for the set of tessellations of a polytope $W \in \mathscr{P}_{d}$. A natural way a local tessellation arises is via restriction to $W$ of a global tessellation. Formally, if $y \in \mathcal{T}$ and $W \in \mathscr{P}_{d}$, we define such a restriction by

$$
y \wedge W:=\{z \cap W: z \in y, \operatorname{dim}(z \cap W)=d\} \in \mathcal{T}_{W},
$$

where $\operatorname{dim}(z \cap W)$ denotes the dimension of the polytope $z \cap W$.

Next, we supply $\mathcal{T}$ and $\mathcal{T}_{W}$ with suitable $\sigma$-fields. For this, we recall that the vague topology on $\mathcal{T}$ is the topology on $\mathcal{T}$ induced by functions of the form

$$
\mathcal{T} \rightarrow \mathbb{R}, \quad y \mapsto \sum_{z \in y} g(z),
$$

where $g: \mathscr{P}_{d} \rightarrow \mathbb{R}$ is any non-negative measurable and bounded function that has compact support, see Kallenberg (2002, Theorem A2.3). Now, we let $\mathfrak{B}(\mathcal{T})$ be the Borel $\sigma$-field generated by the vague topology on $\mathcal{T}$. For $W \in \mathscr{P}_{d}$ and $\mathcal{T}_{W}$ let $\mathfrak{B}\left(\mathcal{T}_{W}\right)$ be defined analogously. One can check that the restriction map $y \mapsto y \wedge W$ then becomes measurable.

Iteration of tessellations. In order to define this operation formally, let $y=\left\{z_{i}\right.$ : $i \in \mathbb{N}\}$ be a tessellation of $\mathbb{R}^{d}$ and $\vec{y}=\left(y^{(i)}\right)_{i \in \mathbb{N}}$ be a sequence of tessellations. Then the tessellation $y \boxplus \vec{y}$, referred to as the iteration of $y$ and $\vec{y}$, is specified by

$$
y \boxplus \vec{y}:=\left\{y^{(i)} \wedge z_{i}: i \in \mathbb{N}\right\} .
$$

Thus, for each $i \in \mathbb{N}$ we restrict the tessellation $y^{(i)}$ to the cell $z_{i} \in y$. This yields a local tessellation of $z_{i}$ and the union of all these local tessellations clearly forms a tessellation of $\mathbb{R}^{d}$. We notice that $\boxplus$ defines a measurable operation from $\mathcal{T} \times \mathcal{T}^{\mathbb{N}}$ to $\mathcal{T}$.

2.4. The local STIT tessellation process. The random STIT tessellation process is denoted by $\underline{Y}$ and its state at time $t$ by $Y_{t}$. Informally, the dynamics of the continuous time random local STIT tessellation process $\underline{Y} \wedge W=\left(Y_{t} \wedge W\right)_{t>0}$ can be described as follows. At time zero, the cell $W$ of the initial tessellation $\{W\}$ receives an exponentially distributed random lifetime with parameter $\Lambda([W])$. When the lifetime of $W$ runs out, a random hyperplane $h \in[W]$ is selected according to the probability distribution $\Lambda_{W}$, given in (2.2), and splits $W$ into the two daughter cells $W \cap h^{+}$and $W \cap h^{-}$. These two daughter cells evolve independently according 
to the same rules, i.e. they receive conditionally independent (given $h$ ) exponentially distributed random lifetimes with parameters $\Lambda\left(\left[W \cap h^{+}\right]\right)$and $\Lambda\left(\left[W \cap h^{-}\right]\right)$, respectively, and then they are divided by random hyperplanes with laws $\Lambda_{W \cap h^{+}}$ and $\Lambda_{W \cap h^{-}}$, respectively, and so on. The cells in $W$ that arise at time $t$ form the local STIT tessellation $Y_{t} \wedge W$.

To describe the construction formally, let $W \in \mathscr{P}_{d}$ be a polytope and $\Lambda$ be a hyperplane measure defined in (2.1). For a tessellation $y \in \mathcal{T}_{W}$, a cell $z \in y$ and a hyperplane $h \in[z] \backslash \mathcal{H}_{0}$ we define the splitting operation $\oslash_{z, h}: \mathcal{T}_{W} \rightarrow \mathcal{T}_{W}$ by

$$
\oslash_{z, h}(y):=(y \backslash\{z\}) \cup\left\{z \cap h^{+}, z \cap h^{-}\right\} .
$$

In other words, $\oslash_{z, h}(y)$ is the tessellation that arises from $y$ by splitting the cell $z$ by means of the hyperplane $h$. The splitting operation is measurable and extends to global tessellations $y \in \mathcal{T}$ as well.

By the local STIT tessellation process $\left(Y_{t} \wedge W\right)_{t \geq 0}$ in $W$ driven by the hyperplane measure $\Lambda$ we understand the continuous time pure jump Markov process on $\mathcal{T}_{W}$ with initial tessellation $Y_{0} \wedge W=W$ and generator

$$
\mathbb{L} g(y):=\sum_{z \in y} \int_{[z]}\left[g\left(\oslash_{z, h}(y)\right)-g(y)\right] \Lambda(\mathrm{d} h), \quad y \in \mathcal{T}_{W}
$$

for all non-negative measurable $g: \mathcal{T}_{W} \rightarrow \mathbb{R}$.

The description of the STIT tessellation process indicates that within each single cell the first division follows the same rules as it does in the cells of a Poisson hyperplane tessellation generated by a space-time hyperplane process $\left(\tilde{\Pi}_{t}\right)_{t>0}$ as given in (4.1). This suggests that a Mecke-type formula can be shown for single divisions of cells. But notice that a maximal polytope, which arises when a cell is divided, depends on the past and (in contrast to the Poisson hyperplane process) it has an impact on the continuation of the STIT process in the future. This is expressed in Theorem 3.1.

2.5. The global STIT tessellation process. So far we have described the STIT tessellation process locally within polytopes $W \in \mathscr{P}_{d}$. However, there exists also a 'global' construction of a STIT tessellation process in $\mathbb{R}^{d}$. Since this construction is rather involved and is needed only as a technical device in our proofs, we decided to postpone its description to Section 4 below. For the moment it is sufficient to confirm that such a process exists. For this, we recall from Nagel and Weiß (2005) the following consistency property. Given two polytopes $W, W^{\prime} \in \mathscr{P}_{d}$ with $W^{\prime} \subset W$, the law of $\left(Y_{t} \wedge W\right) \wedge W^{\prime}$ coincides with that of $Y_{t} \wedge W^{\prime}$, where, recall, for a tessellation $y \in \mathcal{T}$ and a polytope $W \in \mathscr{P}_{d}, y \wedge W$ stands for the restriction of $y$ to $W$. For all $t>0$, this consistency property together with the consistency theorem for random closed sets, see Schneider and Weil (2008, Theorem 2.3.1), yield the existence of a random tessellation $Y_{t}$ with the property that its restriction to any $W \in \mathscr{P}_{d}$ has the same distribution as the previously constructed local STIT tessellation $Y_{t} \wedge W$. The translation invariance of the hyperplane measure $\Lambda$ also ensures that the law of $Y_{t}$ is invariant under translations. One can also show that consistency extends to the finite-dimensional distributions of the processes $\underline{Y} \wedge W=\left(Y_{t} \wedge W\right)_{t>0}$. This way, the classical Kolmogorov extension theorem ensures the existence of a global STIT tessellation process $\underline{Y}=\left(Y_{t}\right)_{t>0}$ with the appropriate finite-dimensional distributions. 
We will use the notation $\underline{Y}=\left(Y_{t}\right)_{t>0}$ for the random STIT tessellation process, $Y_{t}$ for its state at time $t>0$. Respective realizations are denoted by $y$ and $y_{t}$. The distribution of $\left(Y_{t}\right)_{t>0}$ is written $\mathbb{P}_{\underline{Y}}$, and correspondingly the distributions of the other random objects. Furthermore, for a tessellation $y_{t}$ denote $\partial y_{t}=\bigcup_{z \in y_{t}} \partial z$.

The global STIT tessellation process enjoys the following fundamental properties (proven in Nagel and Weiß, 2005):

STIT scaling: The dilation $t Y_{t}$ of $Y_{t}$ by factor $t$ has the same distribution as $Y_{1}$, the STIT tessellation with time parameter 1 , that is,

$$
t Y_{t} \stackrel{D}{=} Y_{1} \quad \text { for all } \quad t>0
$$

where multiplication of a tessellation $y \in \mathcal{T}$ with a factor $t$ stands for the transformation $t y=\{t z: z \in y\}$ and $t z=\{t x: x \in z\}$, see Lemma 5 in Nagel and Weißs (2005).

STIT property: Stability of distribution under the operation of iteration of tessellations.

Let $\underline{Y}=\left(Y_{t}\right)_{t>0}$ be a STIT tessellation process driven by some hyperplane measure $\Lambda$ as in (2.1), and let $\underline{\underline{Y}}=\left(\underline{Y}^{(i)}\right)_{i \in \mathbb{N}}$ be a sequence of i.i.d. copies of $\underline{Y}$. For fixed $s>0$, we write $\vec{Y}_{s}=\left(Y_{s}{ }^{(i)}\right)_{i \in \mathbb{N}}$. Then

$$
Y_{t} \stackrel{D}{=} Y_{s} \boxplus \vec{Y}_{t-s} \quad \text { for all } \quad 0<s<t,
$$

cf. Nagel and Weiß (2005, Lemma 2). This implies in particular that $Y_{2 t} \stackrel{D}{=} Y_{t} \boxplus \vec{Y}_{t}$ for all $t>0$. The STIT property means that

$$
Y_{t} \stackrel{D}{=} 2\left(Y_{t} \boxplus \vec{Y}_{t}\right) \quad \text { for all } \quad t>0 .
$$

STIT intersections: The intersection of the STIT tessellation $Y_{t}$ with a line $L=$ span $u$, where $u \in \mathcal{S}_{+}^{d-1}$ (upper unit half-sphere) is a Poisson point process with intensity $t \Lambda([u])$ (here $u$ has to be interpreted as the line segment connecting the origin with $u$ ).

2.6. The process of maximal polytopes. As described above, any extant cell $z$ in a STIT tessellation has a random lifetime, and at the end of its lifetime, at time $s$ say, it is divided by a hyperplane $h$. Then we call $(p, s) \in \mathscr{P}_{d-1} \times(0, \infty)$ with $p=z \cap h$ a maximal $(d-1)$-polytope, marked with its birth time $s$.

We emphasize that after its birth, a maximal $(d-1)$-polytope can be intersected by other maximal $(d-1)$-polytopes and thus be subdivided further, independently in both of the half-spaces generated by $h$, i.e., in the two cells adjacent to the maximal polytope. But regardless of such events, it will be referred to as a birth time marked maximal polytope, at all times after its birth.

For any $t>0$ we denote by $M_{t}=M_{t}\left(Y_{t}\right)=\sum_{(p, s) \in M, s<t} \delta_{(p, s)}$ the point process of all birth time marked maximal $(d-1)$-polytopes of the global STIT tessellation $Y_{t}$. Thus $M_{t}$ is a point process on the product space $\mathscr{P}_{d-1} \times(0, \infty)$, i.e., it is a random variable with values in $\mathcal{N}\left(\mathscr{P}_{d-1} \times(0, \infty)\right)$, the set of locally finite counting measures on $\mathscr{P}_{d-1} \times(0, \infty)$, supplied with the Borel $\sigma$-field $\mathfrak{B}\left(\mathcal{N}\left(\mathscr{P}_{d-1} \times(0, \infty)\right)\right)$ induced by the vague topology. As usual, we write $(p, s) \in M_{t}$ if $M_{t}(\{(p, s)\})>0$.

By $M=M\left(\left(Y_{t}\right)_{t>0}\right)$ we denote the random point process of birth time marked maximal $(d-1)$-polytopes pertaining to the STIT process $\underline{Y}=\left(Y_{t}\right)_{t>0}$. Also $M$ is a point process on the state space $\mathscr{P}_{d-1} \times(0, \infty)$. 
We emphasize that, given a realization $m$ of a birth time marked maximal polytope process, one can uniquely reconstruct the trajectory $y(m)=\left(y\left(m_{t}\right)\right)_{t>0}$ of a STIT tessellation process that has $m$ as the realization of the pertaining maximal $(d-1)$-polytope process.

We consider the $k$-dimensional faces of maximal $(d-1)$-polytopes, and we refer to them as maximal $k$-polytopes, $k=0, \ldots, d-2$. They appear as the intersection of a sequence of $d-k$ maximal polytopes of dimension $d-1$. It is important to note that for dimensions $d \geq 3$ not all intersections of $d-k$ maximal polytopes (even if the intersection has dimension $k$ ) are faces of maximal polytopes. To see this, consider e.g. three maximal $(d-1)$-polytopes $p_{1}, p_{2}, p_{3}$ such that $p_{1} \cap p_{2} \cap p_{3} \neq \emptyset$, and $p_{2}, p_{3}$ are located in different half-spaces generated by the hyperplane containing $p_{1}$. In view of this, the polytopes which generate a maximal $k$-polytope have to fulfill additional conditions, which will be formalized in the proof of Proposition 3.2.

\section{Results}

3.1. A Mecke-type formula for STIT tessellations. For a realization $m$ of the birth time marked process $M$ of maximal $(d-1)$-polytopes we use the notation $m_{(+t)}:=$ $\{(p, s+t):(p, s) \in m\}$, to express a time shift by $t$ of all the birth times. Furthermore, for $(p, s) \in m$ we denote by $z(p, s) \in y(m)$ the uniquely determined cell in the trajectory $y(m)$ that is divided at time $s$ by the maximal polytope $p$. Finally, for a cell $z \in \overline{\mathscr{P}}_{d}$ denote by

$$
m \wedge z=\{(p \cap z, s):(p, s) \in m, p \cap \stackrel{\circ}{z} \neq \emptyset\},
$$

the restriction of $m$ to $z$.

We are now prepared to present the first main result of this paper, that may be regarded as a Mecke-type formula for STIT tessellations as discussed in some detail after its statement. We postpone the proof to Section 4 below.

Theorem 3.1. Let $M$ be the process of birth time marked maximal (d-1)-polytopes of a (global) STIT tessellation process $\left(Y_{t}\right)_{t>0}$ driven by a hyperplane measure $\Lambda$. Let $\mathbb{P}_{M}$ be the distribution of $M$ and $\mathbb{P}_{Y_{s}}$ be that of $Y_{s}$ at time $s>0$. Then

$$
\begin{gathered}
\int \sum_{(p, s) \in m} g(m \wedge z(p, s), z(p, s), p, s) \mathbb{P}_{M}(\mathrm{~d} m) \\
=\iint \sum_{z \in y_{s}} \int\left[\iint g\left((z \cap h) \cup\left(m_{(+s)}^{(1)} \wedge\left(z \cap h^{+}\right)\right) \cup\left(m_{(+s)}^{(2)} \wedge\left(z \cap h^{-}\right)\right), z, z \cap h, s\right)\right. \\
\left.\mathbb{P}_{M}\left(\mathrm{~d} m^{(1)}\right) \mathbb{P}_{M}\left(\mathrm{~d} m^{(2)}\right)\right] \Lambda_{z}(\mathrm{~d} h) \Lambda([z]) \mathbb{P}_{Y_{s}}\left(\mathrm{~d} y_{s}\right) \mathrm{d} s
\end{gathered}
$$

for all non-negative measurable functions $g: \mathcal{N}\left(\mathscr{P}_{d-1} \times(0, \infty)\right) \times \mathscr{P}_{d} \times \mathscr{P}_{d-1} \times$ $(0, \infty) \rightarrow \mathbb{R}$

Theorem 3.1 shares some similarities with the Mecke formula for Poisson point processes. To re-phrase the latter, let $\Gamma$ be a Poisson point process with $\sigma$-finite intensity measure $\mu$ and distribution $\mathbb{P}_{\Gamma}$ on a measurable space $[\mathcal{E}, \mathfrak{B}(\mathcal{E})]$. Then

$$
\int \sum_{x \in \gamma} g(x, \gamma) \mathbb{P}_{\Gamma}(\mathrm{d} \gamma)=\iint g\left(x, \gamma+\delta_{x}\right) \mu(\mathrm{d} x) \mathbb{P}_{\Gamma}(\mathrm{d} \gamma)
$$


for all non-negative measurable functions $g: \mathcal{E} \times \mathcal{N}(\mathcal{E}) \rightarrow \mathbb{R}$, see Chapter 4 in Last and Penrose $(2017+)$. Obviously, $M$ is not a Poisson point process, but formally, the left-hand side of (3.1) has the same structure as the left-hand side of the Mecke formula for Poisson point processes. Moreover, on the right-hand side of (3.1) we see that an additional hyperplane $h$ is introduced at time $s$ (applying the intensity measure $\left.\Lambda_{z}(\mathrm{~d} h) \Lambda([z]) \mathrm{d} s\right)$, which is similar to the right-hand side of the Mecke formula. The main differences are that in (3.1) for each cell $z \in y_{s}$ a hyperplane is added and moreover, after the division of a cell $z$ by a hyperplane $h$, realizations (denoted $m^{(1)}$ and $\left.m^{(2)}\right)$ of independent copies of $M$ are needed to continue the process in time.

It is also interesting to compare our Theorem 3.1 with Theorem 3.1 in Georgii et al. (2015), where the class of so-called branching random tessellation has been investigated. These tessellation processes constitute a far reaching generalization of the concept of STIT tessellation processes and allow, in particular, for the interaction of cells during the random cell division process as well as for marks (colours) attached to the cells that are also allowed to influence the cell splitting mechanism. Specialized to our context, this result says that for any fixed $t>0$,

$$
\begin{aligned}
& \int \sum_{(p, s) \in m_{t}} g\left(\left(y\left(m_{r}\right)\right)_{r<s}, z(p, s), p, s\right) \mathbf{1}\{s \leq t\} \mathbb{P}_{M_{t}}\left(\mathrm{~d} m_{t}\right) \\
& =\iint \sum_{z \in y_{s}}\left[\int g\left(\left(y_{r}\right)_{r \leq s}, z, z \cap h, s\right) \Lambda_{z}(\mathrm{~d} h) \mathbf{1}\{s \leq t\}\right] \Lambda([z]) \mathbb{P}_{Y_{s}}\left(\mathrm{~d} y_{s}\right) \mathrm{d} s
\end{aligned}
$$

for all non-negative measurable functions $g:\left\{\mathcal{T}^{(0, s]}, 0<s<t\right\} \times \mathscr{P}_{d} \times \mathscr{P}_{d-1} \times$ $(0, t) \rightarrow \mathbb{R}$

Here, $\mathcal{T}^{(0, s]}$ stands for the class of all measurable mappings from $(0, s]$ to $\mathcal{T}$ which contain the realisations of a STIT tessellation process on the time interval $(0, s]$. We notice that relation (3.3) is confined to a finite time horizon for technical reasons. Another significant difference is that in (3.3) the functions $g$ are allowed to depend on the evolution that took place in the past of a given time $s$ only (this is reflected by the appearance of $\left.\left(y_{r}\right)_{r \leq s}\right)$. In contrast, the function $g$ in Theorem 3.1 above can depend on a potentially infinite time horizon, including the evolution after the birth of a particular maximal polytope. On the other hand, relation (3.3) allows for functions that do not only depend on the tessellation within the cell in which a maximal polytope is born, but also on its surrounding (and the colors attached to the cells within this surrounding).

3.2. Application to maximal polytopes. For a realization $m$ of the marked point process $M$ of maximal $(d-1)$-polytopes and for $k=0, \ldots, d-1$, let

$$
\left(\left(p_{1}, s_{1}\right), \ldots,\left(p_{d-k}, s_{d-k}\right)\right) \in m^{d-k}
$$

denote a tuple of maximal polytopes together with their birth times. We denote such a tuple by $(\mathbf{p}, \mathbf{s}, k) \in m^{d-k}$ if and only if $s_{1}<\ldots<s_{d-k}$ and $\overline{\mathbf{p}}=\bigcap_{i=1}^{d-k} p_{i}$ is a maximal $k$-polytope of the STIT tessellation process. If $k<d-1$ this is a $k$-dimensional face of a maximal $(d-1)$-polytope. In this case we call $(\overline{\mathbf{p}}, \mathbf{s})=$ $\left(\bigcap_{i=1}^{d-k} p_{i}, \mathbf{s}\right)$ a maximal $k$-polytope of the STIT tessellation process, marked with its birth time tuple s. Accordingly, we denote $\mathbf{h}=\left(h_{1}, \ldots h_{d-k}\right) \in \mathcal{H}^{d-k}$ and 
$\overline{\mathbf{h}}=\bigcap_{i=1}^{d-k} h_{i}$. If we write $(\mathbf{p}, \mathbf{s}, k) \in m_{t}^{d-k}$ we mean that $(\mathbf{p}, \mathbf{s}, k) \in m^{d-k}$ and that $s_{d-k}<t$.

In the following proposition we consider, for a fixed time parameter $t>0$ and a fixed dimension $k \leq d-1$, the set of all birth time marked maximal $k$-polytopes $(\overline{\mathbf{p}}, \mathbf{s})$ and the trace of the STIT tessellation on them, that is, the intersection

$$
m_{t} \sqcap \overline{\mathbf{p}}:=\left(m_{t} \backslash\left\{\left(p_{1}, s_{1}\right), \ldots,\left(p_{d-k}, s_{d-k}\right)\right\}\right) \cap \overline{\mathbf{p}}
$$

of $\overline{\mathbf{p}}$ with the other maximal $(d-1)$-polytopes of $m_{t}$. Note that $m_{t} \sqcap \overline{\mathbf{p}}$ describes the tessellation structure induced by $m_{t}$ in the (relative) interior of the maximal $k$-polytope $\overline{\mathbf{p}}$.

Proposition 3.2. For $t>0$ and for all non-negative measurable functions $g$ : $\mathscr{P}_{k} \times(0, t)^{d-k} \times \mathfrak{B}\left(\mathbb{R}^{d}\right) \rightarrow \mathbb{R}$,

$$
\begin{gathered}
\int \sum_{(\mathbf{p}, \mathbf{s}, k) \in m_{t}^{d-k}} g\left(\overline{\mathbf{p}}, \mathbf{s}, m_{t} \sqcap \overline{\mathbf{p}}\right) \mathbb{P}_{M_{t}}\left(\mathrm{~d} m_{t}\right) \\
=2^{d-k-1} \int \ldots \sum_{z \in y_{s_{d-k}}} g\left(z \cap \overline{\mathbf{h}}, \mathbf{s}, z \cap \overline{\mathbf{h}} \cap\left[\bigcup_{i=1}^{d-k-1} \partial y_{t-s_{i}}^{(i)} \cup \partial y_{t-s_{d-k}}^{+} \cup \partial y_{t-s_{d-k}}^{-}\right]\right) \\
\mathbb{P}_{\underline{Y}}^{\otimes(d-k+1)}\left(\mathrm{d}\left(\underline{y}^{(1)}, \ldots, \underline{y}^{(d-k-1)}, \underline{y}^{+}, \underline{y}^{-}\right)\right) \\
\cdot \mathbf{1}\{z \cap \overline{\mathbf{h}} \neq \emptyset\} \Lambda^{\otimes(d-k)}(\mathrm{d} \mathbf{h}) \mathbb{P}_{Y_{s_{d-k}}}\left(\mathrm{~d} y_{s_{d-k}}\right) \\
\cdot \mathbf{1}\left\{0<s_{1}<\ldots<s_{d-k}<t\right\} \mathrm{d} s_{1} \ldots \mathrm{d} s_{d-k} .
\end{gathered}
$$

If the function $g$ in Proposition 3.2 depends on the birth time marked maximal $k$-polytope only, then the restriction to a fixed time $t>0$ can be omitted, and the result can be modified as follows.

Corollary 3.3. For all non-negative measurable functions $g: \mathscr{P}_{k} \times(0, \infty)^{d-k} \rightarrow \mathbb{R}$,

$$
\begin{gathered}
\int \sum_{(\mathbf{p}, \mathbf{s}, k) \in m^{d-k}} g(\overline{\mathbf{p}}, \mathbf{s}) \mathbb{P}_{M}(\mathrm{~d} m) \\
=2^{d-k-1} \int \ldots \int \sum_{z \in y_{s_{d-k}}} g(z \cap \overline{\mathbf{h}}, \mathbf{s}) \mathbf{1}\{z \cap \overline{\mathbf{h}} \neq \emptyset\} \Lambda^{\otimes(d-k)}(\mathrm{d} \mathbf{h}) \mathbb{P}_{Y_{s_{d-k}}}\left(\mathrm{~d} y_{s_{d-k}}\right) \\
\cdot \mathbf{1}\left\{0<s_{1}<\ldots<s_{d-k}\right\} \mathrm{d} s_{1} \ldots \mathrm{d} s_{d-k} .
\end{gathered}
$$

3.3. Densities and distributions of typical polytopes. In this section we introduce densities of intrinsic volumes and distributions of typical weighted maximal polytopes. These notions as well as the auxiliary Lemma 3.4 will be used later to prove results for maximal polytopes.

For a fixed $k \in\{0, \ldots, d-1\}$ and a fixed time $t>0$, let us consider the marked point process $\Phi_{t}$ of circumcenters of maximal $k$-polytopes of the STIT tessellation $Y_{t}$, which we mark with the maximal $(d-1)$-polytopes and their birth times and with the 'internal structure' of the maximal $k$-polytopes induced by $M_{t}$.

For a polytope $q$ denote by $c(q)$ its circumcenter and define the mapping

$$
m_{t} \mapsto\left\{\left(c(\overline{\mathbf{p}}), \overline{\mathbf{p}}-c(\overline{\mathbf{p}}), \mathbf{s}, m_{t} \sqcap \overline{\mathbf{p}}\right):(\mathbf{p}, \mathbf{s}, k) \in m_{t}^{d-k}\right\} .
$$


By $\mathbb{P}_{\Phi_{t}}$ we denote the image measure of $\mathbb{P}_{M_{t}}$ under this mapping. Next, we define

$$
\varrho_{k, t}^{(j)}:=\frac{1}{\ell_{d}(B)} \int \sum_{(x, q, \mathbf{s}, T) \in \varphi_{t}} \mathbf{1}\{x \in B\} V_{j}(q) \mathbb{P}_{\Phi_{t}}\left(\mathrm{~d} \varphi_{t}\right)
$$

for $B \in \mathfrak{B}\left(\mathbb{R}^{d}\right)$ with $0<\ell_{d}(B)<\infty, t>0, k \in\{0, \ldots, d-1\}$, and $V_{j}$ is the $j$ th intrinsic volume, $j \in\{0, \ldots, k\}$. (Notice that the value in (3.6) does not depend on the choice of $B$, see e.g. Schneider and Weil, 2008, Theorem 4.1.3).

In particular, $\varrho_{k, t}^{(0)}$ is the intensity of the point process of circumcenters of maximal $k$-polytopes and in general, $\varrho_{k, t}^{(j)}$ is the mean cumulative (or total) $j$ th intrinsic volume of all maximal $k$-polytopes per unit volume.

Campbell's Theorem, see Schneider and Weil (2008, Theorem 3.5.3), implies that the probability measure $\mathbb{P}_{\Phi_{t}}$ can be disintegrated, that is, there exists a probability measure $\mathbb{Q}_{(\overline{\mathbf{P}}, \boldsymbol{\beta}, \tau), t}$ such that the Palm formula

$$
\begin{aligned}
& \int \sum_{(x, q, \mathbf{s}, T) \in \varphi_{t}} g(x, q, \mathbf{s}, T) \mathbb{P}_{\Phi_{t}}\left(\mathrm{~d} \varphi_{t}\right) \\
& =\varrho_{k, t}^{(0)} \iint g(x, q, \mathbf{s}, T) \mathbb{Q}_{(\overline{\mathbf{P}}, \boldsymbol{\beta}, \tau), t}(\mathrm{~d}(q, \mathbf{s}, T)) \ell_{d}(\mathrm{~d} x)
\end{aligned}
$$

holds for all non-negative measurable functions $g: \mathbb{R}^{d} \times \mathscr{P}_{k} \times(0, t)^{d-k} \times \mathfrak{B}\left(\mathbb{R}^{d}\right) \rightarrow \mathbb{R}$. A random $k$-dimensional polytope of $\mathscr{P}_{k}$ (endowed with the tuple of its birth times and the internal structure on it) with distribution $\mathbb{Q}_{(\overline{\mathbf{P}}, \boldsymbol{\beta}, \tau), t}$ is called a typical maximal $k$-polytope of the tessellation $Y_{t}$.

In what follows, we also consider typical weighted maximal $k$-polytopes of $Y_{t}$, with the intrinsic volumes $V_{j}, 0 \leq j \leq k$, as weights. Their distribution $\mathbb{Q}_{(\overline{\mathbf{P}}, \boldsymbol{\beta}, \tau), t}^{(j)}$ is defined by the weighted Palm formula

$$
\begin{gathered}
{\left[\int V_{j}(q) \mathbb{Q}_{\overline{\mathbf{P}}, t}(\mathrm{~d} q)\right]^{-1} \int \sum_{(x, q, \mathbf{s}, T) \in \varphi_{t}} V_{j}(q) g(x, q, \mathbf{s}, T) \mathbb{P}_{\Phi_{t}}\left(\mathrm{~d} \varphi_{t}\right)} \\
=\varrho_{k, t}^{(0)} \iint g(x, q, \mathbf{s}, T) \mathbb{Q}_{(\overline{\mathbf{P}}, \boldsymbol{\beta}, \tau), t}^{(j)}(\mathrm{d}(q, \mathbf{s}, T)) \ell_{d}(\mathrm{~d} x)
\end{gathered}
$$

where $\mathbb{Q}_{\overline{\mathbf{P}}, t}$ is the marginal distribution of $\mathbb{Q}_{(\overline{\mathbf{P}}, \boldsymbol{\beta}, \tau), t}$ for $\overline{\mathbf{P}}$. Note that $\mathbb{Q}_{(\overline{\mathbf{P}}, \boldsymbol{\beta}, \tau), t}^{(0)}=$ $\mathbb{Q}_{(\overline{\mathbf{P}}, \boldsymbol{\beta}, \tau), t}$.

Combining (3.6) and (3.8) immediately leads to the identity

$$
\varrho_{k, t}^{(j)}=\varrho_{k, t}^{(0)} \int V_{j}(q) \mathbb{Q}_{\overline{\mathbf{P}}, t}(\mathrm{~d} q) .
$$

Moreover, using the scaling property (2.4) of STIT tessellations and the homogeneity of the intrinsic volumes, one easily checks that for $t>0, k \in\{0, \ldots, d-1\}$ and $j \in\{0, \ldots, k\}$,

$$
\varrho_{k, t}^{(j)}=t^{d-j} \varrho_{k, 1}^{(j)}
$$

Since $\mathbb{P}_{\Phi_{t}}$ is the image measure of $\mathbb{P}_{M_{t}}$ under the mapping (3.5), the left-hand side of (3.8) can be transformed accordingly. This yields the following lemma. 
Lemma 3.4. For $d \geq 2, k \in\{0, \ldots, d-1\}, g: \mathscr{P}_{k} \times(0, t)^{d-k} \times \mathfrak{B}\left(\mathbb{R}^{d}\right) \rightarrow \mathbb{R}$ non-negative and measurable, and $t>0$,

$$
\begin{aligned}
& \varrho_{k, t}^{(j)} \int g(q, \mathbf{s}, T) \mathbb{Q}_{(\overline{\mathbf{p}}, \boldsymbol{\beta}, \tau), t}^{(j)}(\mathrm{d}(q, \mathbf{s}, T)) \\
= & \int \sum_{(\mathbf{p}, \mathbf{s}, k) \in m_{t}^{d-k}} V_{j}(\overline{\mathbf{p}}) \mathbf{1}\left\{c(\overline{\mathbf{p}}) \in[0,1]^{d}\right\} g\left(\overline{\mathbf{p}}-c(\overline{\mathbf{p}}), \mathbf{s},\left(m_{t} \sqcap \overline{\mathbf{p}}\right)-c(\overline{\mathbf{p}})\right) \mathbb{P}_{M_{t}}\left(\mathrm{~d} m_{t}\right) .
\end{aligned}
$$

Proposition 3.2 or Corollary 3.3 together with an integration with respect to the time coordinates $s_{1}, \ldots, s_{d-k-1}$ imply that $\varrho_{k, t}^{(j)}$ can be represented as

$$
\begin{aligned}
\varrho_{k, t}^{(j)}= & 2^{d-k-1} \frac{1}{\ell_{d}(B)} \iiint \sum_{z \in y_{s_{d-k}}} \mathbf{1}\{c(z \cap \overline{\mathbf{h}}) \in B\} V_{j}(z \cap \overline{\mathbf{h}}) \\
& \mathbb{P}_{Y_{s_{d-k}}}\left(\mathrm{~d} y_{s_{d-k}}\right) \Lambda^{\otimes(d-k)}(\mathrm{d} \mathbf{h}) \mathbf{1}\left\{0<s_{d-k}<t\right\} \frac{s_{d-k}^{d-k-1}}{(d-k-1) !} \mathrm{d} s_{d-k} .
\end{aligned}
$$

It will be useful to have a more concise representation for $\varrho_{k, 1}^{(j)}$, which in view of (3.10) is no restriction of generality.

Proposition 3.5. For all $B \in \mathfrak{B}\left(\mathbb{R}^{d}\right)$ with $0<\ell_{d}(B)<\infty, k \in\{0, \ldots, d-1\}$ and $j \in\{0, \ldots, k\}$ one has that

$$
\begin{aligned}
\varrho_{k, 1}^{(j)}=2^{d-k-1} \frac{1}{(d-k-1) !(d-j)} \cdot \frac{1}{\ell_{d}(B)} \\
\cdot \iint \sum_{z \in y_{1}} \mathbf{1}\{c(z \cap \overline{\mathbf{h}}) \in B\} V_{j}(z \cap \overline{\mathbf{h}}) \Lambda^{\otimes(d-k)}(\mathrm{d} \mathbf{h}) \mathbb{P}_{Y_{1}}\left(\mathrm{~d} y_{1}\right) .
\end{aligned}
$$

3.4. Markov properties of typical maximal polytopes and their birth time distributions. We are now going to apply Proposition 3.2 to prove the Markov properties for the joint birth time distribution of the typical maximal $k$-polytope. We start by determining the marginal distribution $\mathbb{Q}_{\boldsymbol{\beta}, t}^{(j)}$, that is, the birth time distribution of the typical $V_{j}$-weighted maximal $k$-polytope of $Y_{t}$. Our next proposition largely extends and unifies earlier results for the special case $k=d-1$ and $j=0$ in Schreiber and Thäle (2013a) and $d=3, k=1$ and $j \in\{0,1\}$ in Thäle et al. (2012).

Theorem 3.6. Let $d \geq 2, k \in\{0, \ldots, d-1\}, j \in\{0, \ldots, k\}$ and $t>0$. The distribution $\mathbb{Q}_{\boldsymbol{\beta}, t}^{(j)}$ of the birth times $\boldsymbol{\beta}=\left(\beta_{1}, \ldots, \beta_{d-k}\right)$ of the typical $V_{j}$-weighted maximal $k$-polytope has the density

$$
\left(s_{1}, \ldots, s_{d-k}\right) \mapsto(d-j)(d-k-1) ! \frac{s_{d-k}^{k-j}}{t^{d-j}} \mathbf{1}\left\{0<s_{1}<\ldots<s_{d-k}<t\right\}
$$

with respect to the Lebesgue measure on $\mathbb{R}^{d-k}$.

After this preparation, the following results can be shown by direct computations.

Corollary 3.7. Let $d \geq 2, k \in\{0, \ldots, d-1\}$ and $j \in\{0, \ldots, k\}$.

(a) The marginal distribution $\mathbb{Q}_{\beta_{d-k}, t}^{(j)}$ of the last birth time of the typical $V_{j^{-}}$ weighted maximal $k$-polytope has the density

$$
s_{d-k} \mapsto(d-j) \frac{s_{d-k}^{d-j-1}}{t^{d-j}} \mathbf{1}\left\{0<s_{d-k}<t\right\}
$$


with respect to the Lebesgue measure on $\mathbb{R}$.

(b) For all $s_{d-k}<t$, the conditional distribution $\mathbb{Q}_{\left(\beta_{1}, \ldots, \beta_{d-k-1}\right), t \mid \beta_{d-k}=s_{d-k}}^{(j)}$ of the birth times $\left(\beta_{1}, \ldots, \beta_{d-k-1}\right)$ of the typical $V_{j}$-weighted maximal $k$ polytope, given $\beta_{d-k}=s_{d-k}$ has the density

$$
\left(s_{1}, \ldots, s_{d-k-1}\right) \mapsto(d-k-1) ! s_{d-k}^{-(d-k-1)} \mathbf{1}\left\{0<s_{1}<\ldots<s_{d-k}\right\}
$$

with respect to the Lebesgue measure on $\mathbb{R}^{d-k-1}$. In particular, this conditional distribution does not depend on $j$, and it is the uniform distribution on the $(d-k-1)$-simplex $\left\{\left(s_{1}, \ldots, s_{d-k-1}\right) \in \mathbb{R}^{d-k-1}: 0<s_{1}<\ldots<\right.$ $\left.s_{d-k-1}<s_{d-k}\right\}$.

Furthermore, the marginal distribution $\mathbb{Q}_{\left(\overline{\mathbf{P}}, \beta_{d-k}\right), t}$ as well as the conditional distribution $\mathbb{Q} \overline{\mathbf{P}}, t \mid \beta_{d-k}=s_{d-k}$ can be calculated.

Corollary 3.8. Let $d \geq 2, k \in\{0, \ldots, d-1\}, j \in\{0, \ldots, k\}, g: \mathscr{P}_{k} \times(0, t) \rightarrow \mathbb{R}$ be non-negative and measurable and $t>0$. Then,

$$
\begin{aligned}
& \int g\left(q, s_{d-k}\right) \mathbb{Q}_{\left(\overline{\mathbf{P}}, \beta_{d-k}\right), t}^{(j)}\left(\mathrm{d}\left(q, s_{d-k}\right)\right) \\
& =2^{d-k-1}\left[\varrho_{k, t}^{(j)}\right]^{-1} t^{d-j} \\
& \left.\quad \iiint \sum_{z \in y_{s_{d-k}}} V_{j}(z \cap \overline{\mathbf{h}}) \cdot \mathbf{1}\left\{c(z \cap \overline{\mathbf{h}}) \in[0,1]^{d}\right\}\right) \cdot g\left((z \cap \overline{\mathbf{h}})-c(z \cap \overline{\mathbf{h}}), s_{d-k}\right) \\
& \Lambda^{\otimes(d-k)}(\mathrm{d} \mathbf{h}) \mathbb{P}_{Y_{s_{d-k}}}\left(\mathrm{~d} y_{s_{d-k}}\right) \frac{s_{d-k}^{-(k-j)}}{(d-k-1) !(d-j)} \mathbb{Q}_{\beta_{d-k}, t}^{(j)}\left(\mathrm{d} s_{d-k}\right) .
\end{aligned}
$$

In particular, for almost all $s_{d-k} \in(0, t)$ the conditional distribution $\mathbb{Q}_{\overline{\mathbf{P}}, t \mid \beta_{d-k}=s_{d-k}}^{(j)}$ is given by

$$
\begin{aligned}
& \int \tilde{g}(q) \mathbb{Q}_{\overline{\mathbf{P}}, t \mid \beta_{d-k}=s_{d-k}}^{(j)}(\mathrm{d} q) \\
& =2^{d-k-1}\left[\varrho_{k, t}^{(j)}\right]^{-1} \frac{s_{d-k}^{-(k-j)}}{(d-k-1) !(d-j)} t^{d-j} \iiint \sum_{z \in y_{s_{d-k}}} V_{j}(z \cap \overline{\mathbf{h}}) \\
& \quad \cdot \mathbf{1}\left\{c(z \cap \overline{\mathbf{h}}) \in[0,1]^{d}\right\} \cdot \tilde{g}((z \cap \overline{\mathbf{h}})-c(z \cap \overline{\mathbf{h}})) \Lambda^{\otimes(d-k)}(\mathrm{d} \mathbf{h}) \mathbb{P}_{Y_{s_{d-k}}}\left(\mathrm{~d} y_{s_{d-k}}\right)
\end{aligned}
$$

for all non-negative and measurable $\tilde{g}: \mathscr{P}_{k} \rightarrow \mathbb{R}$.

An application of the result obtained so far yields the following conditional independence property, which can also be interpreted as a Markov

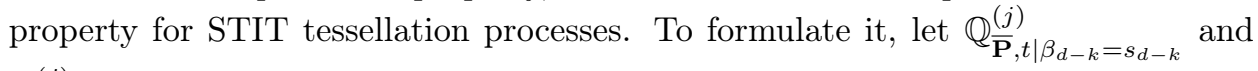
$\mathbb{Q}_{\left(\beta_{1}, \ldots, \beta_{d-k-1}\right), t \mid \beta_{d-k}=s_{d-k}}^{(j)}$ denote conditional distributions (as indicated by their indexes), pertaining to $\mathbb{Q}_{(\overline{\mathbf{P}}, \boldsymbol{\beta}), t}^{(j)}$.

Theorem 3.9. Let $d \geq 2, k \in\{0, \ldots, d-1\}, j \in\{0, \ldots, k\}, g: \mathscr{P}_{k} \times(0, t)^{d-k} \rightarrow \mathbb{R}$ be non-negative and measurable and $t>0$. Then,

$$
\int g(q, \mathbf{s}) \mathbb{Q}_{\left(\overline{\mathbf{P}}, \beta_{1}, \ldots, \beta_{d-k}\right), t}^{(j)}(\mathrm{d}(q, \mathbf{s}))=\iiint g(q, \mathbf{s})
$$




$$
\mathbb{Q}_{\overline{\mathbf{P}}, t \mid \beta_{d-k}=s_{d-k}}^{(j)}(\mathrm{d} q) \mathbb{Q}_{\left(\beta_{1}, \ldots, \beta_{d-k-1}\right), t \mid \beta_{d-k}=s_{d-k}}^{(j)}\left(\mathrm{d}\left(s_{1}, \ldots, s_{d-k-1}\right)\right) \mathbb{Q}_{\beta_{d-k}}^{(j)}\left(\mathrm{d} s_{d-k}\right),
$$

which is equivalent to the conditional independence of the typical $V_{j}$-weighted maximal $k$-polytope $\overline{\mathbf{P}}$ and $\left(\beta_{1}, \ldots, \beta_{d-k-1}\right)$, given the last birth time $\beta_{d-k}=s_{d-k}$.

3.5. The number of internal vertices on maximal segments. Now we turn to an application of the results, considering the maximal segments, i.e., the maximal 1polytopes of the STIT tessellation $Y_{t}$ with a driving measure $\Lambda$ as in (2.1). These segments may have internal vertices (that is, vertices that are located in the relative interior of a segment), which arise already at the time of birth of the segment (when $d \geq 3$ ) and thereafter subject to further subdivision of adjacent cells. In the planar case, a maximal segment is always born without internal vertices. The following theorem provides the distribution of the number of internal points of the typical and the typical length weighted maximal segment, respectively. Formally, for $t>0$, $j=0,1$ and $n=0,1,2, \ldots$ we define for the typical $V_{j}$-weighted maximal 1-polytope with distribution $\mathbb{Q}_{(\overline{\mathbf{P}}, \boldsymbol{\beta}, \tau), t}^{(j)}$

$$
\mathrm{p}_{1, j}(n):=\int \mathbf{1}\{\# T=n\} \mathbb{Q}_{(\overline{\mathbf{P}}, \boldsymbol{\beta}, \tau), t}^{(j)}(\mathrm{d}(q, \mathbf{s}, T)),
$$

where the variable $T$ stands for the 'internal structure' as defined in (3.4).

Theorem 3.10. Let $d \geq 2$. For all $n \in\{0,1,2, \ldots\}$ the probabilities $\mathrm{p}_{1,0}(n)$ and $\mathrm{p}_{1,1}(n)$, respectively, do neither depend on $\Lambda$ nor on $t$, and they are given by

$\mathrm{p}_{1,0}(n)=d(d-2) ! \int_{0}^{1} \int_{0}^{s_{d-1}} \ldots \int_{0}^{s_{2}} \frac{s_{d-1}^{2}\left(d-2 s_{d-1}-s_{d-2}-\ldots-s_{1}\right)^{n}}{\left(d-s_{d-1}-s_{d-2}-\ldots-s_{1}\right)^{n+1}} \mathrm{~d} s_{1} \ldots \mathrm{d} s_{d-1}$

and

$$
\mathrm{p}_{1,1}(n)
$$

$=(n+1)(d-1) ! \int_{0}^{1} \int_{0}^{s_{d-1}} \ldots \int_{0}^{s_{2}} \frac{s_{d-1}^{2}\left(d-2 s_{d-1}-s_{d-2}-\ldots-s_{1}\right)^{n}}{\left(d-s_{d-1}-s_{d-2}-\ldots-s_{1}\right)^{n+2}} \mathrm{~d} s_{1} \ldots \mathrm{d} s_{d-1}$.

The fact that $\mathrm{p}_{1,0}(n)$ and $\mathrm{p}_{1,1}(n)$ do not depend on the time $t$ is consistent with the scaling property (2.4) of a STIT tessellation, because the number of internal vertices on a maximal 1-polytope does not change when the tessellation is rescaled. Theorem 3.10 can also be used to compute the moments of the respective distributions. The following identities are readily checked by using Theorem 3.10.

Corollary 3.11. Let $d \geq 2$ and $N_{d}^{(j)}, j=0,1$, be random variables with distributions given by $\mathrm{p}_{1, j}$. Then

$$
\mathbb{E} N_{d}^{(0)}=\frac{1}{2} \frac{d^{2}-d+2}{d-1} \quad \text { and } \quad \mathbb{E} N_{d}^{(1)}= \begin{cases}+\infty & \text { if } d=2 \\ \frac{d^{2}-2 d+4}{d-2} & \text { if } d \geq 3 .\end{cases}
$$

Note that $\mathbb{E} N_{2}^{(0)}=\mathbb{E} N_{3}^{(0)}$, while $\mathbb{E} N_{d}^{(0)}$ is strictly increasing for all $d \geq 3$. In contrast, for the mean number of internal vertices on the typical length-weighted maximal segment we have that $\mathbb{E} N_{3}^{(1)}=7, \mathbb{E} N_{4}^{(1)}=6, \mathbb{E} N_{5}^{(1)}=6 \frac{1}{3}$ and $\mathbb{E} N_{6}^{(1)}=7$ and, considered as a function of $d, \mathbb{E} N_{d}^{(1)}$ is strictly increasing for $d \geq 5$.

In the planar case $d=2$, as mentioned above, the probabilities $\mathrm{p}_{1,0}(n)$ are known from Mecke et al. (2011); Thäle (2010), whereas for $d=3$ the formula for $\mathrm{p}_{1,0}(n)$ 
has been established in Thäle et al. (2012) by different methods. Our approach in the present paper is more general and allows to deduce the corresponding formula also for the length-weighted maximal segment as well as to deal with arbitrary space dimensions. As a concrete example, take $d=3$ and consider the length-weighted typical maximal segment. Here, we have

$$
\begin{aligned}
& \mathrm{p}_{1,1}(0)=5+18 \ln 2-\frac{63}{4} \ln 3 \approx 0.173506, \\
& \mathrm{p}_{1,1}(1)=28+90 \ln 2-\frac{657}{8} \ln 3 \approx 0.159712, \quad \text { etc. }
\end{aligned}
$$

The mean number of internal vertices is 7 in this case. The values $p_{1,1}(n)$ may be determined from the formula in Theorem 3.10 by straightforward integration.

\section{Proofs}

4.1. A global construction. The main technical device in the proof of Theorem 3.1 is a global construction developed in Mecke et al. (2008a,b, 2011) for the STIT tessellation process with driving measure $\Lambda$. Here we summarize the essential ingredients that are needed for our later purposes.

We start with a Poisson point process $\Pi$ on the measurable space $[\mathcal{H} \times(0, \infty)$, $\mathfrak{B}(\mathcal{H} \times(0, \infty))]$ with the intensity measure $\Lambda \otimes \ell_{+}$. Now, we define the random process $\left(\tilde{\Pi}_{t}\right)_{t>0}$ of marked Poisson hyperplane processes, putting

$$
\tilde{\Pi}_{t}:=\{(h, s) \in \Pi: s \leq t\}, \quad t>0 .
$$

For $(h, s) \in \Pi$ we interpret $s$ as the birth time of the hyperplane $h$ and write $\beta(h)=s$.

Our assumption on the measure $\Lambda$ ensures that for all $t>0$, the Poisson hyperplane process $\Pi_{t}=\left\{h \in \mathcal{H}:(h, s) \in \tilde{\Pi}_{t}\right\}$ a.s. (almost surely) induces a tessellation of $\mathbb{R}^{d}$. We denote this Poisson hyperplane tessellation by $X_{t}$, and by $\underline{X}=\left(X_{t}\right)_{t>0}$ the corresponding random process. For any $t>0$ there is an a.s. uniquely determined random cell $Z_{t}^{0}$ of the Poisson hyperplane tessellation $X_{t}$ that contains the origin. The random process on $\mathscr{P}_{d}$ of these zero cells is denoted by $\left(Z_{t}^{0}\right)_{t>0}$. Clearly, this process is a pure jump process. Let $\left(\eta_{k}\right)_{k \in \mathbb{Z}}$ be the sequence of its jump times with the convention that $\eta_{1}<1 \leq \eta_{2}$. In Mecke et al. (2008a, Lemma 4.1) it was shown that

$$
\bigcup_{k \in \mathbb{Z}} Z_{\eta_{k}}^{0}=\mathbb{R}^{d}
$$

At each jump time $\eta_{k}$ a cell $\hat{Z}_{k}$ is chopped off from the current zero cell. The basic idea is to start immediately within each of these new cells $\hat{Z}_{k}$ a local STIT tessellation process as described in Section 2.4 (with the window $W$ replaced by $\hat{Z}_{k}$ ). This can formally be described as follows.

Let $\Sigma^{*}$ be a Poisson point process on the measurable space

$$
\left[\mathbb{R}^{d} \times(-\infty, 0) \times \mathcal{N}(\mathcal{H} \times(0, \infty)), \mathfrak{B}\left(\mathbb{R}^{d} \times(-\infty, 0) \times \mathcal{N}(\mathcal{H} \times(0, \infty))\right)\right]
$$

with intensity measure $\ell_{d} \times \ell_{-} \times \mathbb{P}_{\Pi}$, where $\mathbb{P}_{\Pi}$ is the distribution of the process $\Pi$ defined above. Further, define $\Sigma:=\Sigma^{*}+\delta_{(0,0, \Pi)}$, where we suppose that the point processes $\Sigma^{*}$ and $\Pi$ are independent. We interpret the points of $\Sigma$ as a collection of random points in $\mathbb{R}^{d}$ that are marked with priorities in $(-\infty, 0)$ and a birth time 
marked hyperplane process from $\mathcal{N}(\mathcal{H} \times(0, \infty))$, the space of locally finite counting measures on $\mathcal{H} \times(0, \infty)$.

The points from $\mathbb{R}^{d} \times(-\infty, 0]$ are designed to select a hyperplane process which is then used for the division of an extant cell. Namely, given a cell $z \in \mathscr{P}_{d}$ we choose the point $(X(z), R(z), \Psi(z)) \in \Sigma$ such that $X(z) \in z$ and $R(z)=\max \{r \in$ $(-\infty, 0]:(x, r, \psi) \in \Sigma, x \in z\}$.

In other words, $X(z)$ is the a.s. uniquely determined point in $z$ with the highest priority. Note that after the first division of $z$ this point remains a.s. the same for one of the two daughter cells, while for the other daughter cell a new point is selected. It is clear that if $z$ is the zero cell we always have that $(X(z), R(z), \Psi(z))=$ $(0,0, \Pi)$. Now, if a cell $z$ is born at time $\beta(z)$ by division of its mother cell or by separating from the current zero cell, and if $(X(z), R(z), \Psi(z))$ is chosen as described, then the marked hyperplane $(h, s) \in \Psi(z)$ is used to divide $z$ further if and only if $h \in[z]$ and $s=\min \left\{s^{\prime}>\beta(z):\left(h^{\prime}, s^{\prime}\right) \in \Psi(z), h^{\prime} \in[z]\right\}$. This further division then leads to the birth time marked maximal $(d-1)$-polytope $(z \cap h, s)$, i.e. $\beta(z \cap h)=s$, and the two new daughter cells have the birth time $s$ as well.

The construction we have described defines a random process on the space $\mathcal{T}$ of tessellations. In fact, it has been shown in Mecke et al. (2008a,b, 2011) that, restricted to a polytope $W$, this process coincides with the local STIT tessellation process in $W$ driven by the hyperplane measure $\Lambda$. As explained in Subsection 2.5, the distribution of this process must then coincide with that one of the global STIT tessellation process $\underline{Y}=\left(Y_{t}\right)_{t>0}$ defined by means of consistency and the Kolmogorov extension theorem. The construction here is an explicit global construction based on the Poisson point process $\Sigma$, and it is the key device in the proof of Theorem 3.1.

4.2. Proof of Theorem 3.1. We are now going to give a proof of Theorem 3.1, which makes use of the global construction outlined in the previous section. We use the same notation as there. Moreover, for a realization $\sigma$ of the Poisson point process $\Sigma$, let $m(\sigma)$ be the uniquely determined realization of the point process of birth time marked maximal $(d-1)$-polytopes. Correspondingly, $\underline{y}(\sigma)$ denotes the realization of the STIT process determined by $\sigma$, and $y(\sigma)_{s}$ its state at time $s$. By $z \in \underline{y}(\sigma)$ we mean any cell which is extant in some time interval (i.e. between its birth and its division) in the realization $y(\sigma)$.

Further, for given $(x, r, \psi) \in \sigma$ and $(h, s) \in \psi$, there can be either no or exactly one cell $z \in y(\sigma)$ such that $z$ is divided by $h$ at time $s$. For better readability we introduce the following abbreviations for a cell $z \in \underline{y}(\sigma)$ and for a given hyperplane $h$ and a time $s$ :

$$
\begin{aligned}
& \mathbf{1}\left(z, \sigma^{*},(x, r, \psi),(h, s)\right) \\
:= & \mathbf{1}\left\{(x, r, \psi) \in \sigma^{*}\right\} \mathbf{1}\{(h, s) \in \psi\} \mathbf{1}\{x \in z, 0 \notin z\} \\
& \cdot \mathbf{1}\left\{r=\max \left\{r^{\prime} \in(-\infty, 0):\left(x^{\prime}, r^{\prime}, \psi^{\prime}\right) \in \sigma^{*}, x^{\prime} \in z\right\}\right\} \\
& \cdot \mathbf{1}\{h \in[z]\} \mathbf{1}\left\{s=\min \left\{s^{\prime}>\beta(z):\left(h^{\prime}, s^{\prime}\right) \in \psi, h^{\prime} \in[z]\right\}\right\}, \\
& \mathbf{1}(z, 0, \pi,(h, s)) \\
:= & \mathbf{1}\{(h, s) \in \pi\} \mathbf{1}\{0 \in z\} \mathbf{1}\{h \in[z]\} \mathbf{1}\left\{s=\min \left\{s^{\prime}>\beta(z):\left(h^{\prime}, s^{\prime}\right) \in \pi\right\}\right\} .
\end{aligned}
$$


The two notations distinguish whether $z$ is a zero-cell or not. The terms are 1 if $z$ is divided by $h$ at time $s$, and 0 otherwise.

At first, because $m(\sigma)$ and $y(\sigma)$ are uniquely determined by $\sigma$, the transformation formula for image measures implies that

$$
\begin{aligned}
A & :=\int \sum_{(p, s) \in m} g(m \wedge z(p, s), z(p, s), p, s) \mathbb{P}_{M}(\mathrm{~d} m) \\
& =\int \sum_{(p, s) \in m(\sigma)} g(m(\sigma) \wedge z(p, s), z(p, s), p, s) \mathbb{P}_{\Sigma}(\mathrm{d} \sigma) .
\end{aligned}
$$

A maximal polytope of dimension $d-1$ appears once a cell gets divided. Using the rules from the global construction of the STIT tessellation, the definition of the process $\Sigma$ as a sum of $\Sigma^{*}$ and $\delta_{(0,0, \Pi)}$ and the abbreviations given above this leads to

$$
\begin{aligned}
A & =\iint \sum_{z \in \underline{y}\left(\sigma^{*}+\delta_{(0,0, \pi)}\right)}\left(\sum_{(x, r, \psi) \in \sigma^{*}} \sum_{(h, s) \in \psi} \mathbf{1}\left(z, \sigma^{*},(x, r, \psi),(h, s)\right)\right. \\
& \left.+\sum_{(h, s) \in \pi} \mathbf{1}(z, 0, \pi,(h, s))\right) g\left(m\left(\sigma^{*}+\delta_{(0,0, \pi)}\right) \wedge z, z, z \cap h, s\right) \mathbb{P}_{\Pi}(\mathrm{d} \pi) \mathbb{P}_{\Sigma^{*}}\left(\mathrm{~d} \sigma^{*}\right) .
\end{aligned}
$$

Applying the Mecke formula (3.2) to the Poisson point processes $\Sigma^{*}$ yields

$$
\begin{aligned}
A & =\iiint \iint_{z \in \underline{y}\left(\sigma^{*}+\delta_{(x, r, \psi)}+\delta_{(0,0, \pi)}\right)}\left(\sum_{(h, s) \in \psi} \mathbf{1}\left(z, \sigma^{*}+\delta_{(x, r, \psi)},(x, r, \psi),(h, s)\right)\right. \\
& \left.+\sum_{(h, s) \in \pi} \mathbf{1}(z, 0, \pi,(h, s))\right) g\left(m\left(\sigma^{*}+\delta_{(x, r, \psi)}+\delta_{(0,0, \pi)}\right) \wedge z, z, z \cap h, s\right) \\
& \mathbb{P}_{\Pi(}(\mathrm{d} \psi) \ell_{d}(\mathrm{~d} x) \ell_{-}(\mathrm{d} r) \mathbb{P}_{\Pi}(\mathrm{d} \pi) \mathbb{P}_{\Sigma^{*}}\left(\mathrm{~d} \sigma^{*}\right) .
\end{aligned}
$$

Next, we apply the Mecke formula (3.2) again, this time with respect to $\psi$ and to $\pi$, i.e. to the Poisson point process $\Pi$, which has intensity measure $\Lambda \otimes \ell_{+}$. This leads to the equation

$$
A=\iiint \iint\left[A_{1}+A_{2}\right] \mathbb{P}_{\Pi}(\mathrm{d} \psi) \ell_{d}(\mathrm{~d} x) \ell_{-}(\mathrm{d} r) \mathbb{P}_{\Pi}(\mathrm{d} \pi) \mathbb{P}_{\Sigma^{*}}\left(\mathrm{~d} \sigma^{*}\right)
$$

with the terms $A_{1}$ and $A_{2}$ given by

$$
\begin{gathered}
A_{1}:=\iint_{z \in \underline{y}\left(\sigma^{*}+\delta_{\left(x, r, \psi+\delta_{(h, s)}\right)}+\delta_{(0,0, \pi)}\right)} \mathbf{1}\left(z, \sigma^{*}+\delta_{\left(x, r, \psi+\delta_{(h, s)}\right)},\left(x, r, \psi+\delta_{(h, s)}\right),(h, s)\right) \\
\cdot g\left(m\left(\sigma^{*}+\delta_{\left(x, r, \psi+\delta_{(h, s)}\right)}+\delta_{(0,0, \pi)}\right) \wedge z, z, z \cap h, s\right) \Lambda(\mathrm{d} h) \mathrm{d} s
\end{gathered}
$$

and

$$
\begin{aligned}
A_{2}:=\iint_{z \in \underline{y}\left(\sigma^{*}+\delta_{(x, r, \psi)}+\delta_{\left(0,0, \pi+\delta_{(h, s)}\right)}\right.} \mathbf{1}\left(z, 0, \pi+\delta_{(h, s)},(h, s)\right) \\
\cdot g\left(m\left(\sigma^{*}+\delta_{(x, r, \psi)}+\delta_{\left(0,0, \pi+\delta_{(h, s)}\right)}\right) \wedge z, z, z \cap h, s\right) \Lambda(\mathrm{d} h) \mathrm{d} s .
\end{aligned}
$$

Now, notice that the $(x, r)$-value of $\left(x, r, \psi+\delta_{(h, s)}\right)$ is the same as that of $(x, r, \psi)$. Furthermore, $z \in \underline{y}\left(\sigma^{*}+\delta_{\left(x, r, \psi+\delta_{(h, s)}\right)}+\delta_{(0,0, \pi)}\right)$ and the value of the indicator 
$\mathbf{1}\left(z, \sigma^{*}+\delta_{\left(x, r, \psi+\delta_{(h, s)}\right)},\left(x, r, \psi+\delta_{(h, s)}\right),(h, s)\right)$ in $A_{1}$ is 1 , if and only if $z \in y\left(\sigma^{*}+\right.$ $\left.\delta_{(x, r, \psi)}+\delta_{(0,0, \pi)}\right)_{s}$ and $z$ is divided by $h$ at time $s$.

If $s>0, z \in y(\sigma)_{s}$ and $h \in[z]$, then let $\underline{y}\left(\sigma, \oslash_{s, z, h}\right)$ denote the realization of the STIT tessellation process which until time $s$ coincides with $\underline{y}(\sigma)$, at time $s$ the cell $z$ is divided by $h$, and after time $s$ the global construction is continued based on $\sigma$. Note that the division of $z$ by $h$ has an impact on the construction after time $s$. With this notation, it follows that

$$
\begin{aligned}
A_{1}=\iint_{z \in y\left(\sigma^{*}+\delta_{(x, r, \psi)}+\delta_{(0,0, \pi)}\right)_{s}} \mathbf{1}\{h \in[z]\} \\
\quad \cdot g\left(m\left(\left(\underline{y}\left(\sigma^{*}+\delta_{(x, r, \psi)}+\delta_{(0,0, \pi)}, \oslash_{s, z, h}\right)\right) \wedge z, z, z \cap h, s\right) \Lambda(\mathrm{d} h) \mathrm{d} s\right.
\end{aligned}
$$

and

$$
\begin{aligned}
A_{2}=\iint & \sum_{z \in y\left(\sigma^{*}+\delta_{(x, r, \psi)}+\delta_{(0,0, \pi)}\right)_{s}} \mathbf{1}\{h \in[z]\} \\
& \cdot g\left(m\left(\left(\underline{y}\left(\sigma^{*}+\delta_{(x, r, \psi)}+\delta_{(0,0, \pi)}, \oslash_{s, z, h}\right)\right) \wedge z, z, z \cap h, s\right) \Lambda(\mathrm{d} h) \mathrm{d} s .\right.
\end{aligned}
$$

Plugging this into (4.2) and applying then the Mecke formula (3.2) (reading it from right to left) to the Poisson point processes $\Sigma^{*}$ (not to $\Pi$ ), we conclude that

$$
\begin{aligned}
A=\int & \int\left[\int \sum_{z \in y\left(\sigma^{*}+\delta_{(0,0, \pi)}\right)_{s}} \sum_{(x, r, \psi) \in \sigma^{*}} \mathbf{1}\{h \in[z]\}\right. \\
& \left.\cdot g\left(m\left(\left(\underline{y}\left(\sigma^{*}+\delta_{(0,0, \pi)}, \oslash_{s, z, h}\right)\right)\right) \wedge z, z, z \cap h, s\right) \Lambda(\mathrm{d} h) \mathrm{d} s\right] \mathbb{P}_{\Pi}(\mathrm{d} \pi) \mathbb{P}_{\Sigma^{*}}\left(\mathrm{~d} \sigma^{*}\right) .
\end{aligned}
$$

Now we use once more the transformation theorem for image measures and the fact that $m$ and $y$ are uniquely determined by $\pi$ and $\sigma^{*}$. Moreover, notice that the cell $z$ is divided for the first time at $s$ using the hyperplane $h$ into two daughter cells and that within these two daughter cells two independent STIT tessellation processes are realized. This yields

$$
\begin{gathered}
A=\iiint \iint \sum_{z \in y_{s}} g\left((z \cap h) \cup\left(m_{(+s)}^{(1)} \wedge\left(z \cap h^{+}\right)\right) \cup\left(m_{(+s)}^{(2)} \wedge\left(z \cap h^{-}\right)\right), z, z \cap h, s\right) \\
\cdot \mathbf{1}\{h \in[z]\} \Lambda(\mathrm{d} h) \mathbb{P}_{M}\left(\mathrm{~d} m^{(1)}\right) \mathbb{P}_{M}\left(\mathrm{~d} m^{(2)}\right) \mathbb{P}_{Y_{s}}\left(\mathrm{~d} y_{s}\right) \mathrm{d} s .
\end{gathered}
$$

Together with the definition (2.2) of the probability measure $\Lambda_{z}$ this finally leads to the identity

$$
\begin{gathered}
A=\iint \sum_{z \in y_{s}} \int\left[\iint g\left((z \cap h) \cup\left(m_{(+s)}^{(1)} \wedge\left(z \cap h^{+}\right)\right) \cup\left(m_{(+s)}^{(2)} \wedge\left(z \cap h^{-}\right)\right), z, z \cap h, s\right)\right. \\
\left.\mathbb{P}_{M}\left(\mathrm{~d} m^{(1)}\right) \mathbb{P}_{M}\left(\mathrm{~d} m^{(2)}\right)\right] \Lambda_{z}(\mathrm{~d} h) \Lambda([z]) \mathbb{P}_{Y_{s}}\left(\mathrm{~d} y_{s}\right) \mathrm{d} s
\end{gathered}
$$

and the proof of the theorem is complete.

4.3. Proof of Proposition 3.2. The purpose of the present subsection is to prove Proposition 3.2. This is prepared by the following technical lemma. Let $\mathcal{F}_{d-1}(z)$ denote the set of all facets (that is, faces of dimension $d-1$ ) of a polytope $z \in \mathscr{P}_{d}$. 
Lemma 4.1. For all non-negative measurable functions $\tilde{g}: \mathscr{P}_{d-1} \rightarrow \mathbb{R}$ and $0<$ $s_{1}<s_{2}$, we have that

$$
\begin{aligned}
& \int \sum_{z_{1} \in y_{s_{1}}} \iint \sum_{z_{2} \in y_{s_{2}-s_{1}} \wedge\left(z_{1} \cap h_{1}^{+}\right)} \tilde{g}\left(z_{1} \cap h_{1} \cap z_{2}\right) \mathbf{1}\left\{\left(z_{2} \cap h_{1}\right) \in \mathcal{F}_{d-1}\left(z_{2}\right)\right\} \\
\mathbf{\cdot 1}\left\{h_{1} \in\left[z_{1}\right]\right\} \mathbb{P}_{Y_{s_{2}-s_{1}}}\left(\mathrm{~d} y_{s_{2}-s_{1}}\right) \Lambda\left(\mathrm{d} h_{1}\right) \mathbb{P}_{Y_{s_{1}}}\left(\mathrm{~d} y_{s_{1}}\right) & \int \sum_{z \in y_{s_{2}}} \int \tilde{g}\left(z \cap h_{1}\right) \mathbf{1}\left\{h_{1} \in[z]\right\} \Lambda\left(\mathrm{d} h_{1}\right) \mathbb{P}_{Y_{s_{2}}}\left(\mathrm{~d} y_{s_{2}}\right) .
\end{aligned}
$$

Proof: Assume that $h_{1} \cap \stackrel{\circ}{z}_{1} \neq \emptyset$ and $z_{2} \in y_{s_{2}-s_{1}} \wedge\left(z_{1} \cap h_{1}^{+}\right)$. Then $z_{2} \cap h_{1} \in \mathcal{F}_{d-1}\left(z_{2}\right)$ if and only if $z_{2} \subset z_{1}$ and there is a cell $z \in y_{s_{2}-s_{1}} \wedge z_{1}$ such that $z_{2}=z \cap h_{1}^{+}$and $z_{2} \cap h_{1}=z \cap h_{1} \neq \emptyset$. Hence, using Fubini's theorem,

$$
\begin{aligned}
& \int \sum_{z_{1} \in y_{s_{1}}} \iint \sum_{z_{2} \in y_{s_{2}-s_{1}} \wedge\left(z_{1} \cap h_{1}^{+}\right)} \tilde{g}\left(z_{1} \cap h_{1} \cap z_{2}\right) \mathbf{1}\left\{\left(z_{2} \cap h_{1}\right) \in \mathcal{F}_{d-1}\left(z_{2}\right)\right\} \\
& \cdot \mathbf{1}\left\{h_{1} \in\left[z_{1}\right]\right\} \mathbb{P}_{Y_{s_{2}-s_{1}}}\left(\mathrm{~d} y_{s_{2}-s_{1}}\right) \Lambda\left(\mathrm{d} h_{1}\right) \mathbb{P}_{Y_{s_{1}}}\left(\mathrm{~d} y_{s_{1}}\right) \\
& =\iiint \sum_{z_{1} \in y_{s_{1}}} \sum_{z \in y_{s_{2}-s_{1}} \wedge z_{1}} \tilde{g}\left(z \cap h_{1}\right) \mathbf{1}\left\{h_{1} \in[z]\right\} \Lambda\left(\mathrm{d} h_{1}\right) \\
& \mathbb{P}_{Y_{s_{2}-s_{1}}}\left(\mathrm{~d} y_{s_{2}-s_{1}}\right) \mathbb{P}_{Y_{s_{1}}}\left(\mathrm{~d} y_{s_{1}}\right) \\
& =\int \sum_{z \in y_{s_{2}}} \int \tilde{g}\left(z \cap h_{1}\right) \mathbf{1}\left\{h_{1} \in[z]\right\} \Lambda\left(\mathrm{d} h_{1}\right) \mathbb{P}_{Y_{s_{2}}}\left(\mathrm{~d} y_{s_{2}}\right) \text {, }
\end{aligned}
$$

where the last equality follows from (2.5).

Now we prove Proposition 3.2. If $(\overline{\mathbf{p}}, \mathbf{s})=\left(\bigcap_{i=1}^{d-k} p_{i}, \mathbf{s}\right)$ is a marked maximal $k$ polytope generated by a $(d-k)$-tuple $\left(\left(p_{1}, s_{1}\right), \ldots\left(p_{d-k}, s_{d-k}\right)\right)=(\mathbf{p}, \mathbf{s}, k) \in m_{t}^{d-k}$, then we can represent it in the following way which will be used in the formulas below. The $(d-1)$-polytope $p_{1}$ is located on a hyperplane $h_{1}$ with birth time $s_{1}$, and at that time it divides a cell $z_{1}$, i.e., $p_{1}=z_{1} \cap h_{1}$. For a STIT tessellation process, on both cells (indicated by + and - ) adjacent to $p_{1}$ appear independent traces until time $s_{2}$ and these two traces will be treated separately. Let us consider the case that the remaining maximal polytopes $\left(\left(p_{2}, s_{2}\right), \ldots\left(p_{d-k}, s_{d-k}\right)\right)$ are located in the cell $z_{1} \cap p_{1}^{+}$. This cell is subdivided in the time interval $\left(s_{1}, s_{2}\right)$ by $\{(p, s) \in$ $\left.m_{t} \wedge\left(z_{1} \cap p_{1}^{+}\right): s_{1}<s<s_{2}\right\}$. Then, at time $s_{2}$, one of the cells, $z_{2} \subseteq z_{1} \cap p_{1}^{+}$is divided by $\left(p_{2}, s_{2}\right)$, and $\operatorname{dim}\left(p_{1} \cap p_{2}\right)=d-2$. In particular, this means that one of the $(d-1)$-dimensional faces of $z_{2}$ is a subset of $p_{1}$, and this face is divided by $p_{2}$. The maximal $(d-1)$-polytope $p_{2}$ is located on a hyperplane $h_{2}$ with birth time $s_{2}$.

This can now be continued inductively. The combination of the possible choices in each step of the adjacent cells, indicated by + and -, leads to a factor $2^{d-k-1}$. The $(d-k)$-tuple $(\mathbf{p}, \mathbf{s}, k) \in m_{t}^{d-k}$ will be processed step by step, and after separating $\left(p_{1}, s_{1}\right)$ the remaining $(d-k-1)$-tuple is denoted $\left(\mathbf{p}^{\mathbf{1}}, \mathbf{s}^{\mathbf{1}}\right)$, and so on. Further, assume $p_{i} \subset h_{i} \in \mathcal{H}$, i.e., the hyperplane $h_{i}$ supports $p_{i}$.

For $0<s<t$ and $m$ a realization of $M$ denote $m_{(+s, t)}:=\left\{\left(p, s^{\prime}+s\right):\left(p, s^{\prime}\right) \in\right.$ $\left.M, 0<s^{\prime}<t-s\right\}$, that is, the set of all birth time marked maximal $(d-1)$ polytopes, with a birth time shifted by $s$, and such that the shifted birth time is between $s$ and $t$. Furthermore, we denote $\overline{\mathbf{p}}^{j}:=\bigcap_{i=j+1}^{d-k} p_{i}$, for $j=1, \ldots d-k-2$. 
Now,

$$
\begin{aligned}
A & :=\int \sum_{(\mathbf{p}, \mathbf{s}, k) \in m_{t}^{d-k}} g\left(\overline{\mathbf{p}}, \mathbf{s}, m_{t} \sqcap \overline{\mathbf{p}}\right) \mathbb{P}_{M_{t}}\left(\mathrm{~d} m_{t}\right) \\
& =\int \sum_{\left(p_{1}, s_{1}\right) \in m_{t}} \sum_{\left(\mathbf{p}^{1}, \mathbf{s}^{1}\right)} g\left(\overline{\mathbf{p}}, \mathbf{s}, m_{t} \sqcap \overline{\mathbf{p}}\right) \mathbb{P}_{M_{t}}\left(\mathrm{~d} m_{t}\right) .
\end{aligned}
$$

Next, we apply Theorem 3.1, exchange the order of integration and partition the sum into two parts. This yields

$$
\begin{aligned}
& A=\iiint \iint \sum_{z_{1} \in y_{s_{1}}}\left[\sum_{\left(\mathbf{p}^{1}, \mathbf{s}^{1}\right) \in\left(m_{\left(+s_{1}, t\right)}^{+,(d-k-1)} \wedge\left(z_{1} \cap h_{1}^{+}\right)\right)} \mathbf{1}\left\{\operatorname{dim}\left(z_{1} \cap h_{1} \cap \overline{\mathbf{p}}^{1}\right)=k\right\}\right. \\
& \cdot g\left(z_{1} \cap h_{1} \cap \overline{\mathbf{p}}^{1}, \mathbf{s},\left[\left(m_{\left(+s_{1}, t\right)}^{-} \cup m_{\left(+s_{1}, t\right)}^{+}\right) \backslash\left\{p_{2}, \ldots, p_{d-k-1}\right\}\right] \cap z_{1} \cap h_{1} \cap \overline{\mathbf{p}}^{1}\right)
\end{aligned}
$$

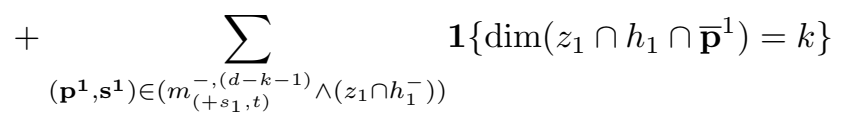

$$
\begin{aligned}
& \left.\cdot g\left(z_{1} \cap h_{1} \cap \overline{\mathbf{p}}^{1}, \mathbf{s},\left[\left(m_{\left(+s_{1}, t\right)}^{-} \cup m_{\left(+s_{1}, t\right)}^{+}\right) \backslash\left\{p_{2}, \ldots, p_{d-k-1}\right\}\right] \cap z_{1} \cap h_{1} \cap \overline{\mathbf{p}}^{1}\right)\right] \\
& \mathbb{P}_{M}\left(\mathrm{~d} m^{-}\right) \mathbb{P}_{M}\left(\mathrm{~d} m^{+}\right) \mathbf{1}\left\{h_{1} \in\left[z_{1}\right]\right\} \Lambda\left(\mathrm{d} h_{1}\right) \mathbf{1}\left\{0<s_{1}<t\right\} P_{Y_{s_{1}}}\left(\mathrm{~d} y_{s_{1}}\right) \mathrm{d} s_{1} .
\end{aligned}
$$

In the first item in squared brackets, i.e., the case $\left(\mathbf{p}^{\mathbf{1}}, \mathbf{s}^{\mathbf{1}}\right) \in\left(m_{\left(+s_{1}, t\right)}^{+,(d-k-1)} \wedge\left(z_{1} \cap\right.\right.$ $\left.h_{1}^{+}\right)$), decompose $\left(\mathbf{p}^{\mathbf{1}}, \mathbf{s}^{\mathbf{1}}\right)$ into $\left(p_{2}, s_{2}\right)$ and the remaining $(d-k-2)$-tuple $\left(\mathbf{p}^{\mathbf{2}}, \mathbf{s}^{\mathbf{2}}\right)$. Applying Theorem 3.1 once again, but this time to

$$
\int \sum_{\left(p_{2}, s_{2}\right) \in\left(m_{\left(+s_{1}, t\right)}^{+} \wedge\left(z_{1} \cap h_{1}^{+}\right)\right)}\{\ldots\} \mathbb{P}_{M}\left(\mathrm{~d} m^{+}\right)
$$

and noting that $z_{2} \subset z_{1}$, yields

$$
\begin{aligned}
& \iint \sum_{\left(\mathbf{p}^{1}, \mathbf{s}^{1}\right) \in\left(m_{\left(+s_{1}, t\right)}^{+,(d-k-1)} \wedge\left(z_{1} \cap h_{1}^{+}\right)\right)} \mathbf{1}\left\{\operatorname{dim}\left(\overline{\mathbf{p}}^{1} \cap z_{1} \cap h_{1}\right)=k\right\} \\
& \cdot g\left(z_{1} \cap h_{1} \cap \overline{\mathbf{p}}^{1}, \mathbf{s},\right. \\
& \left.\left[\left(m_{\left(+s_{1}, t\right)}^{-} \cup m_{\left(+s_{1}, t\right)}^{+}\right) \backslash\left\{z_{1} \cap h_{1}, p_{2}, \ldots, p_{d-k-1}\right\}\right] \cap z_{1} \cap h_{1} \cap \overline{\mathbf{p}}^{1}\right) \\
& \mathbb{P}_{M}\left(\mathrm{~d} m^{-}\right) \mathbb{P}_{M}\left(\mathrm{~d} m^{+}\right) \\
& =\int \ldots \int \sum_{z_{2} \in y_{s_{2}-s_{1}} \wedge\left(z_{1} \cap h_{1}^{+}\right)}\left[\sum_{\left(\mathbf{p}^{2}, \mathbf{s}^{2}\right) \in\left(m_{\left(+s_{2}, t\right)}^{++,(d-k-2)} \wedge\left(z_{2} \cap h_{2}^{+}\right)\right)}\right. \\
& \mathbf{1}\left\{\operatorname{dim}\left(\overline{\mathbf{p}}^{2} \cap z_{2} \cap h_{1} \cap h_{2}\right)=k\right\} \\
& \cdot g\left(z_{2} \cap h_{1} \cap h_{2} \cap \overline{\mathbf{p}}^{2}, \mathbf{s},\right. \\
& \left.\left[\left(m_{\left(+s_{1}, t\right)}^{-} \cup m_{\left(+s_{2}, t\right)}^{++} \cup m_{\left(+s_{2}, t\right)}^{+-}\right) \backslash\left\{p_{3}, \ldots, p_{d-k-1}\right\}\right] \cap z_{2} \cap h_{1} \cap h_{2} \cap \overline{\mathbf{p}}^{2}\right)
\end{aligned}
$$




$$
\begin{aligned}
& +\sum_{\left(\mathbf{p}^{2}, \mathbf{s}^{2}\right) \in\left(m_{\left(+s_{2}, t\right)}^{+,,(d-k-2)} \wedge\left(z_{2} \cap h_{2}^{-}\right)\right)} \mathbf{1}\left\{\operatorname{dim}\left(\overline{\mathbf{p}}^{2} \cap z_{2} \cap h_{1} \cap h_{2}\right)=k\right\} \\
& \cdot g\left(z_{2} \cap h_{1} \cap h_{2} \cap \overline{\mathbf{p}}^{2}, \mathbf{s},\right. \\
& \left.\left.\quad\left[\left(m_{\left(+s_{1}, t\right)}^{-} \cup m_{\left(+s_{2}, t\right)}^{++} \cup m_{\left(+s_{2}, t\right)}^{+-}\right) \backslash\left\{p_{3}, \ldots, p_{d-k-1}\right\}\right] \cap z_{2} \cap h_{1} \cap h_{2} \cap \overline{\mathbf{p}}^{2}\right)\right] \\
& \mathbb{P}_{M}\left(\mathrm{~d} m^{-}\right) \mathbb{P}_{M}\left(\mathrm{~d} m^{++}\right) \mathbb{P}_{M}\left(\mathrm{~d} m^{+-}\right) \mathbb{P}_{Y_{s_{2}-s_{1}}}\left(\mathrm{~d} y_{s_{2}-s_{1}}\right) \\
& \mathbf{1}\left\{h_{2} \in\left[z_{2}\right]\right\} \Lambda\left(\mathrm{d} h_{2}\right) \mathbf{1}\left\{0<s_{1}<s_{2}<t\right\} \mathrm{d} s_{2} .
\end{aligned}
$$

Now, we apply this argument repeatedly to all summands and decompose $(\mathbf{p}, \mathbf{s})$ step by step. Note that $z_{d-k} \subset \ldots \subset z_{1}$, and also that the intersections like $\left[\left(m_{\left(+s_{1}, t\right)}^{-} \cup m_{\left(+s_{2}, t\right)}^{++} \cup m_{\left(+s_{2}, t\right)}^{+-}\right) \backslash\left\{p_{3}, \ldots, p_{d-k-1}\right\}\right] \cap z_{2} \cap h_{1} \cap h_{2} \cap \overline{\mathbf{p}}^{2}$ do not depend on the combinations of signs (which determine a part of the space) in the upper index. Hence, Lemma 4.1 yields that $A$ is equal to

$$
\begin{aligned}
A= & \sum_{\left(a_{1}, \ldots, a_{d-k-1}\right) \in\{+,-\}^{d-k-1}} \int \ldots \int \sum_{z_{d-k} \in y_{s_{d-k}}} \\
& \cdot g\left(z_{d-k} \cap \overline{\mathbf{h}}, \mathbf{s},\left(\bigcup_{i=1}^{d-k-1} m_{\left(+s_{i}, t\right)}^{(i)} \cup m_{\left(+s_{d-k}, t\right)}^{+} \cup m_{\left(+s_{d-k}, t\right)}^{-}\right) \cap z_{d-k} \cap \overline{\mathbf{h}}\right) \\
& \cdot \mathbf{1}\left\{z_{d-k} \cap \overline{\mathbf{h}} \neq \emptyset\right\} \Lambda\left(\mathrm{d} h_{d-k}\right) \ldots \Lambda\left(\mathrm{d} h_{2}\right) \Lambda\left(\mathrm{d} h_{1}\right) \\
& \mathbb{P}_{M}\left(\mathrm{~d} m^{+}\right) \mathbb{P}_{M}\left(\mathrm{~d} m^{-}\right) \mathbb{P}_{M}\left(\mathrm{~d} m^{(d-k-1)}\right) \ldots \mathbb{P}_{M}\left(\mathrm{~d} m^{(1)}\right) \mathbb{P}_{Y_{s_{d-k}}}\left(\mathrm{~d} y_{s_{d-k}}\right) \\
& \cdot \mathbf{1}\left\{0<s_{1}<\ldots<s_{d-k}<t\right\} \mathrm{d} s_{1} \ldots \mathrm{d} s_{d-k} .
\end{aligned}
$$

Now substitute $m_{\left(+s_{i}, t\right)}^{(i)}$ by the corresponding STIT tessellations $y_{t-s_{i}}^{(i)}=y\left(m_{\left(+s_{i}, t\right)}^{(i)}\right)$. Furthermore, due to the spatial consistency of STIT tessellations the values of the summands do not depend on $\left(a_{1}, \ldots, a_{d-k-1}\right) \in\{+,-\}^{d-k-1}$. Noting finally, that the first sum is running over $2^{d-k-1}$ terms leads to the identity

$$
\begin{gathered}
\int \sum_{(\mathbf{p}, \mathbf{s}, k) \in m_{t}^{d-k}} g\left(\overline{\mathbf{p}}, \mathbf{s}, m_{t} \sqcap \overline{\mathbf{p}}\right) \mathbb{P}_{M_{t}}\left(\mathrm{~d} m_{t}\right) \\
=2^{d-k-1} \int \ldots \sum_{z \in y_{s_{d-k}}} g\left(z \cap \overline{\mathbf{h}}, \mathbf{s}, z \cap \overline{\mathbf{h}} \cap\left[\bigcup_{i=1}^{d-k-1} \partial y_{t-s_{i}}^{(i)} \cup \partial y_{t-s_{d-k}}^{+} \cup \partial y_{t-s_{d-k}}^{-}\right]\right) \\
\mathbb{P}_{\underline{Y}}^{\otimes(d-k+1)}\left(\mathrm{d}\left(\underline{y}^{(1)}, \ldots \underline{y}^{(d-k-1)}, \underline{y}^{+}, \underline{y}^{-}\right)\right) \mathbf{1}\{z \cap \overline{\mathbf{h}} \neq \emptyset\} \Lambda^{\otimes(d-k)}(\mathrm{d} \mathbf{h}) \\
\mathbb{P}_{Y_{s_{d-k}}}\left(\mathrm{~d} y_{s_{d-k}}\right) \cdot \mathbf{1}\left\{0<s_{1}<\ldots<s_{d-k}<t\right\} \mathrm{d} s_{1} \ldots \mathrm{d} s_{d-k},
\end{gathered}
$$

which completes the proof.

4.4. Proof of Proposition 3.5. Using the scaling property (2.4), changing the order of integration and substituting $y_{s_{d-k}}$ by $\frac{1}{s_{d-k}} y_{1}$, we obtain from (3.12) that

$$
\begin{aligned}
\varrho_{k, 1}^{(j)}= & 2^{d-k-1} \frac{1}{\ell_{d}(B)} \iiint \sum_{z \in y_{s_{d-k}}} \mathbf{1}\{c(z \cap \overline{\mathbf{h}}) \in B\} V_{j}(z \cap \overline{\mathbf{h}}) \\
& \mathbb{P}_{Y_{s_{d-k}}}\left(\mathrm{~d} y_{s_{d-k}}\right) \Lambda^{\otimes(d-k)}(\mathrm{d} \mathbf{h}) \mathbf{1}\left\{0<s_{d-k}<1\right\} \frac{s_{d-k}^{d-k-1}}{(d-k-1) !} \mathrm{d} s_{d-k}
\end{aligned}
$$




$$
\begin{gathered}
=2^{d-k-1} \frac{1}{\ell_{d}(B)} \iiint \sum_{z \in \frac{1}{s_{d-k}} y_{1}} \mathbf{1}\{c(z \cap \overline{\mathbf{h}}) \in B\} V_{j}(z \cap \overline{\mathbf{h}}) \\
\mathbb{P}_{Y_{1}}\left(\mathrm{~d} y_{1}\right) \Lambda^{\otimes(d-k)}(\mathrm{d} \mathbf{h}) \mathbf{1}\left\{0<s_{d-k}<1\right\} \frac{s_{d-k}^{d-k-1}}{(d-k-1) !} \mathrm{d} s_{d-k} \\
=2^{d-k-1} \frac{1}{\ell_{d}(B)} \iiint \sum_{z \in y_{1}} \mathbf{1}\left\{c\left(\frac{1}{s_{d-k}} z \cap \overline{\mathbf{h}}\right) \in B\right\} V_{j}\left(\frac{1}{s_{d-k}} z \cap \overline{\mathbf{h}}\right) \\
\mathbb{P}_{Y_{1}}\left(\mathrm{~d} y_{1}\right) \Lambda^{\otimes(d-k)}(\mathrm{d} \mathbf{h}) \mathbf{1}\left\{0<s_{d-k}<1\right\} \frac{s_{d-k}^{d-k-1}}{(d-k-1) !} \mathrm{d} s_{d-k} .
\end{gathered}
$$

We consider the two inner integrals separately. Let $\gamma_{1}$ denote the mean number of cell centroids per unit volume and let $\mathbb{Q}_{1}$ denote the distribution of the typical cell of $Y_{1}$. Then an application of Campbell's theorem, multiplication with $s_{d-k}$, and the homogeneity of the $j$ th intrinsic volume $V_{j}$ yield

$$
\begin{gathered}
I:=\frac{1}{\ell_{d}(B)} \iint \sum_{z \in y_{1}} \mathbf{1}\left\{c\left(\frac{1}{s_{d-k}} z \cap \overline{\mathbf{h}}\right) \in B\right\} V_{j}\left(\frac{1}{s_{d-k}} z \cap \overline{\mathbf{h}}\right) \mathbb{P}_{Y_{1}}\left(\mathrm{~d} y_{1}\right) \Lambda^{\otimes(d-k)}(\mathrm{d} \mathbf{h}) \\
=\frac{1}{\ell_{d}(B)} \gamma_{1} \iiint \mathbf{1}\left\{c\left((z+x) \cap s_{d-k} \overline{\mathbf{h}}\right) \in s_{d-k} B\right\} s_{d-k}^{-j} \\
\cdot V_{j}\left((z+x) \cap s_{d-k} \overline{\mathbf{h}}\right) \ell_{d}(\mathrm{~d} x) \mathbb{Q}_{1}(\mathrm{~d} z) \Lambda^{\otimes(d-k)}(\mathrm{d} \mathbf{h})
\end{gathered}
$$

In a next step, we use that $s_{d-k} \overline{\mathbf{h}}=\overline{\mathbf{h}}+\left(s_{d-k}-1\right) x^{\perp}=\overline{\mathbf{h}}_{0}+s_{d-k} x^{\perp}$, which is a translation of $\overline{\mathbf{h}}$, where $x^{\perp}=\overline{\mathbf{h}} \cap \overline{\mathbf{h}}_{0}^{\perp}, \overline{\mathbf{h}}_{0}$ the $k$-dimensional linear subspace parallel to $\overline{\mathbf{h}}$ and $\overline{\mathbf{h}}_{0}^{\perp}$ its orthogonal complement. The image of the measure $\mathbf{1}\{\operatorname{dim}(\overline{\mathbf{h}})=$ $k\} \cdot \Lambda^{\otimes(d-k)}(\mathrm{d} \mathbf{h})$ (the product measure, endowed with the indicator density) under the mapping $\mathbf{h} \mapsto \overline{\mathbf{h}}$ is invariant under translations. Then, according to Schneider and Weil (2008, Theorem 4.4.1), we obtain that

$$
\begin{aligned}
I & =\frac{1}{\ell_{d}(B)} \gamma_{1} \iiint \mathbf{1}\left\{c((z+x) \cap \overline{\mathbf{h}}) \in s_{d-k} B\right\} s_{d-k}^{-j} V_{j}((z+x) \cap \overline{\mathbf{h}}) \\
& \ell_{d}(\mathrm{~d} x) \mathbb{Q}_{1}(\mathrm{~d} z) s_{d-k}^{-(d-k)} \Lambda^{\otimes(d-k)}(\mathrm{d} \mathbf{h}) \\
& =s_{d-k}^{d} \frac{1}{\ell_{d}\left(s_{d-k} B\right)} \gamma_{1} \iiint \mathbf{1}\left\{c((z+x) \cap \overline{\mathbf{h}}) \in s_{d-k} B\right\} s_{d-k}^{-j} V_{j}((z+x) \cap \overline{\mathbf{h}}) \\
& \ell_{d}(\mathrm{~d} x) \mathbb{Q}_{1}(\mathrm{~d} z) s_{d-k}^{-(d-k)} \Lambda^{\otimes(d-k)}(\mathrm{d} \mathbf{h}) \\
& =s_{d-k}^{k-j} \frac{1}{\ell_{d}\left(s_{d-k} B\right)} \gamma_{1} \iiint \mathbf{1}\left\{c((z+x) \cap \overline{\mathbf{h}}) \in s_{d-k} B\right\} V_{j}((z+x) \cap \overline{\mathbf{h}}) \\
& =s_{d-k}^{k-j} \frac{1}{\ell_{d}(B)} \iint \sum_{z \in y_{1}} \mathbf{1}\{c(z \cap \overline{\mathbf{h}}) \in B\} V_{j}(z \cap \overline{\mathbf{h}}) \mathbb{P}_{Y_{1}}\left(\mathrm{~d} y_{1}\right) \Lambda^{\otimes(d-k)}(\mathrm{d} \mathbf{h}),
\end{aligned}
$$

where the last equation follows from Campbell's theorem and by replacing $s_{d-k} B$ by $B$.

Plugging this expression for $I$ into the equation for $\varrho_{k, 1}^{(j)}$ above yields 


$$
\begin{aligned}
\varrho_{k, 1}^{(j)}= & 2^{d-k-1} \frac{1}{\ell_{d}(B)} \iiint \sum_{z \in y_{1}} s_{d-k}^{k-j} \mathbf{1}\{c(z \cap \overline{\mathbf{h}}) \in B\} V_{j}(z \cap \overline{\mathbf{h}}) \\
& \quad \mathbb{P}_{Y_{1}}\left(\mathrm{~d} y_{1}\right) \Lambda^{\otimes(d-k)}(\mathrm{d} \mathbf{h}) \mathbf{1}\left\{0<s_{d-k}<1\right\} \frac{s_{d-k}^{d-k-1}}{(d-k-1) !} \mathrm{d} s_{d-k} \\
= & 2^{d-k-1} \frac{1}{(d-k-1) !(d-j)} \frac{1}{\ell_{d}(B)} \\
& \cdot \iint \sum_{z \in y_{1}} \mathbf{1}\{c(z \cap \overline{\mathbf{h}}) \in B\} V_{j}(z \cap \overline{\mathbf{h}}) \mathbb{P}_{Y_{1}}\left(\mathrm{~d} y_{1}\right) \Lambda^{\otimes(d-k)}(\mathrm{d} \mathbf{h}),
\end{aligned}
$$

where the last equation results by integration with respect to $s_{d-k}$.

4.5. Proof of Theorem 3.6. For any non-negative measurable function $g:(0, t)^{d-k} \rightarrow \mathbb{R}$, Corollary 3.3 and an application of Lemma 3.4 yield

$$
\begin{aligned}
& \int g(\mathbf{s}) \mathbb{Q}_{\boldsymbol{\beta}, t}^{(j)}(\mathrm{d} \mathbf{s})=\int g(\mathbf{s}) \mathbb{Q}_{(\overline{\mathbf{P}}, \boldsymbol{\beta}, \tau), t}^{(j)}(\mathrm{d}(q, \mathbf{s}, T)) \\
= & 2^{d-k-1}\left[\varrho_{k, t}^{(j)}\right]^{-1} \int \ldots \sum_{z \in y_{s_{d-k}}} \mathbf{1}\left\{c(z \cap \overline{\mathbf{h}}) \in[0,1]^{d}\right\} \cdot V_{j}(z \cap \overline{\mathbf{h}}) \cdot g(\mathbf{s}) \Lambda^{\otimes(d-k)}(\mathrm{d} \mathbf{h}) \\
\cdot & \mathbf{1}\left\{0<s_{1}<\ldots<s_{d-k}<t\right\} \mathrm{d} s_{1} \ldots \mathrm{d} s_{d-k-1} \mathbb{P}_{Y_{s_{d-k}}}\left(\mathrm{~d} y_{s_{d-k}}\right) \mathrm{d} s_{d-k} .
\end{aligned}
$$

Using (2.4) and substituting $y_{s_{d-k}}$ by $\frac{1}{s_{d-k}} y_{1}$ we obtain (similarly to the calculations in the proof of Proposition 3.5)

$$
\begin{gathered}
\int g(\mathbf{s}) \mathbb{Q}_{\boldsymbol{\beta}, t}^{(j)}(\mathrm{d} \mathbf{s})=2^{d-k-1}\left[\varrho_{k, t}^{(j)}\right]^{-1} \int \ldots \int_{z \in \frac{1}{s_{d-k}} y_{1}} \mathbf{1}\left\{c(z \cap \overline{\mathbf{h}}) \in[0,1]^{d}\right\} \cdot V_{j}(z \cap \overline{\mathbf{h}}) \cdot g(\mathbf{s}) \\
\Lambda^{\otimes(d-k)}(\mathrm{d} \mathbf{h}) \mathbb{P}_{Y_{1}}\left(\mathrm{~d} y_{1}\right) \mathbf{1}\left\{0<s_{1}<\ldots<s_{d-k}<t\right\} \mathrm{d} s_{1} \ldots \mathrm{d} s_{d-k-1} \mathrm{~d} s_{d-k} \\
=2^{d-k-1}\left[\varrho_{k, t}^{(j)}\right]^{-1} \iint \sum_{z \in y_{1}} \mathbf{1}\left\{c(z \cap \overline{\mathbf{h}}) \in[0,1]^{d}\right\} \cdot V_{j}(z \cap \overline{\mathbf{h}}) \Lambda^{\otimes(d-k)}(\mathrm{d} \mathbf{h}) \mathbb{P}_{Y_{1}}\left(\mathrm{~d} y_{1}\right) \\
\cdot \int \ldots \int g(\mathbf{s}) \cdot s_{d-k}^{k-j} \cdot \mathbf{1}\left\{0<s_{1}<\ldots<s_{d-k}<t\right\} \mathrm{d} s_{1} \ldots \mathrm{d} s_{d-k-1} \mathrm{~d} s_{d-k} .
\end{gathered}
$$

We can now use Proposition 3.5 together with the scaling relation (3.10) to evaluate $\varrho_{k, t}^{(j)}$. This yields the desired result immediately.

4.6. Proof of Corollary 3.8. Applying Lemma 3.4, Corollary 3.3 and Corollary 3.7 (a) yields

$$
\begin{aligned}
& \int g\left(q, s_{d-k}\right) \mathbb{Q}_{\left(\overline{\mathbf{P}}, \beta_{d-k}\right), t}^{(j)}\left(\mathrm{d}\left(q, s_{d-k}\right)\right) \\
= & 2^{d-k-1}\left[\varrho_{k, t}^{(j)}\right]^{-1} \\
& \iiint \sum_{z \in y_{s-k}} V_{j}(z \cap \overline{\mathbf{h}}) \cdot \mathbf{1}\left\{c(z \cap \overline{\mathbf{h}}) \in[0,1]^{d}\right\} \cdot g\left((z \cap \overline{\mathbf{h}})-c(z \cap \overline{\mathbf{h}}), s_{d-k}\right) \\
& \Lambda^{\otimes(d-k)}(\mathrm{d} \mathbf{h}) \mathbb{P}_{Y_{s_{d-k}}}\left(\mathrm{~d} y_{s_{d-k}}\right) \frac{s_{d-k}^{d-k-1}}{(d-k-1) !} \mathbf{1}\left\{0<s_{d-k}<t\right\} \mathrm{d} s_{d-k}
\end{aligned}
$$




$$
\begin{aligned}
= & 2^{d-k-1}\left[\varrho_{k, t}^{(j)}\right]^{-1} \\
& \left.\iint \sum_{z \in y_{s_{d-k}}} V_{j}(z \cap \overline{\mathbf{h}}) \cdot \mathbf{1}\left\{c(z \cap \overline{\mathbf{h}}) \in[0,1]^{d}\right\}\right) \cdot g\left((z \cap \overline{\mathbf{h}})-c(z \cap \overline{\mathbf{h}}), s_{d-k}\right) \\
& \Lambda^{\otimes(d-k)}(\mathrm{d} \mathbf{h}) \mathbb{P}_{Y_{s_{d-k}}}\left(\mathrm{~d} y_{s_{d-k}}\right) \frac{s_{d-k}^{-(k-j)}}{(d-k-1) !(d-j)} t^{d-j} \mathbb{Q}_{\beta_{d-k}, t}^{(j)}\left(\mathrm{d} s_{d-k}\right)
\end{aligned}
$$

and the result follows.

4.7. Proof of Theorem 3.9. It is sufficient to consider functions $g$ of the form

$$
g(q, \mathbf{s})=g_{1}(q) \cdot g_{2}\left(s_{1}, \ldots, s_{d-k-1}\right) \cdot g_{3}\left(s_{d-k}\right),
$$

where $g_{1}: \mathscr{P}_{k} \rightarrow \mathbb{R}, g_{2}:(0, t)^{d-k-1} \rightarrow \mathbb{R}, g_{3}:(0, t) \rightarrow \mathbb{R}$ are non-negative measurable functions. The proposition for general $g$ follows then by a standard measure-theoretic procedure. As in the proof of Theorem 3.6, we have

$$
\begin{aligned}
& \int g(q, \mathbf{s}) \mathbb{Q}_{\left(\overline{\mathbf{P}}, \beta_{1}, \ldots, \beta_{d-k}\right), t}^{(j)}(\mathrm{d}(q, \mathbf{s})) \\
= & {\left[\varrho_{k, t}^{(j)}\right]^{-1} \int \sum_{(\mathbf{p}, \mathbf{s}, k) \in m_{t}^{d-k}} \mathbf{1}\left\{c(\overline{\mathbf{p}}) \in[0,1]^{d}\right\} V_{j}(\overline{\mathbf{p}}) g(\overline{\mathbf{p}}-c(\overline{\mathbf{p}}), \mathbf{s}) \mathbb{P}_{M_{t}}\left(\mathrm{~d} m_{t}\right) } \\
= & 2^{d-k-1}\left[\varrho_{k, t}^{(j)}\right]^{-1} \\
& \left.\int \ldots \sum_{z \in y_{s_{d-k}}} g_{1}((z \cap \overline{\mathbf{h}})-c(z \cap \overline{\mathbf{h}}))\right) \mathbf{1}\left\{c(z \cap \overline{\mathbf{h}}) \in[0,1]^{d}\right\} V_{j}(z \cap \overline{\mathbf{h}}) \\
& \Lambda^{\otimes(d-k)}(\mathrm{d} \mathbf{h}) \mathbb{P}_{Y_{s_{d-k}}}\left(\mathrm{~d} y_{s_{d-k}}\right) \\
& \cdot g_{2}\left(s_{1}, \ldots, s_{d-k-1}\right) \mathbf{1}\left\{0<s_{1}<\ldots<s_{d-k}\right\} \mathrm{d} s_{1} \ldots \mathrm{d} s_{d-k-1} \\
& \cdot g_{3}\left(s_{d-k}\right) \mathbf{1}\left\{0<s_{d-k}<t\right\} \mathrm{d} s_{d-k} .
\end{aligned}
$$

Now, we apply Corollary 3.7 and Corollary 3.8 and obtain that this is equal to

$$
\begin{gathered}
\iiint \frac{(d-k-1) !(d-j) s_{d-k}^{k-j}}{t^{d-j}} g_{1}(q) \mathbb{Q}_{\overline{\mathbf{P}}, t \mid \beta_{d-k}=s_{d-k}}^{(j)}(\mathrm{d} q) \\
\cdot g_{2}\left(s_{1}, \ldots, s_{d-k-1}\right) \frac{1}{(d-k-1) !} s_{d-k}^{d-k-1} \mathbb{Q}_{\left(\beta_{1}, \ldots, \beta_{d-k-1}\right), t \mid \beta_{d-k}=s_{d-k}}^{(j)} \\
\mathrm{d}\left(s_{1}, \ldots, s_{d-k-1}\right) g_{3}\left(s_{d-k}\right) \frac{t^{d-j}}{(d-j)} s_{d-k}^{-(d-j-1)} \mathbb{Q}_{\beta_{d-k}}^{(j)}\left(\mathrm{d} s_{d-k}\right) \\
=\iiint g(q, \mathbf{s}) \mathbb{Q}_{\overline{\mathbf{P}}, t \mid \beta_{d-k}=s_{d-k}}^{(j)}(\mathrm{d} q) \mathbb{Q}_{\left(\beta_{1}, \ldots, \beta_{d-k-1}\right), t \mid \beta_{d-k}=s_{d-k}}^{(j)} \\
\mathrm{d}\left(s_{1}, \ldots, s_{d-k-1}\right) \mathbb{Q}_{\beta_{d-k}}^{(j)}\left(\mathrm{d} s_{d-k}\right),
\end{gathered}
$$

which completes the proof.

4.8. Proof of Theorem 3.10. For fixed $\mathbf{h}=\left(h_{1}, \ldots, h_{d-1}\right) \in \mathcal{H}^{d-1}$ with the hyperplanes in general position define the line $\overline{\mathbf{h}}=\bigcap_{i=1}^{d-1} h_{i}$. Because the intersection of a STIT with a line is a Poisson point process (see Nagel and Weiß, 2003), 
$\overline{\mathbf{h}} \cap\left[\bigcup_{i=1}^{d-2} \partial y_{t-s_{i}}^{(i)} \cup \partial y_{t-s_{d-1}}^{+} \cup \partial y_{t-s_{d-1}}^{-}\right]$is a realization of a superposition of $d$ independent Poisson point processes on the line $\overline{\mathbf{h}}$, with a law invariant under translations on this line. Due to the stationarity of STIT tessellations, the intensity of this point process depends only on the direction of this line, which we denote by $u \in \mathcal{S}^{d-1}$, and, up to a factor $b(u)>0$, it is given by the sum

$$
a(\mathbf{s})=\sum_{i=1}^{d-2}\left(t-s_{i}\right)+2\left(t-s_{d-1}\right)=d \cdot t-2 s_{d-1}-\sum_{i=1}^{d-2} s_{i} .
$$

Thus for any cell $z$ the number of points of

$$
z \cap \overline{\mathbf{h}} \cap\left[\bigcup_{i=1}^{d-2} \partial y_{t-s_{i}}^{(i)} \cup \partial y_{t-s_{d-1}}^{+} \cup \partial y_{t-s_{d-1}}^{-}\right]
$$

follows a Poisson distribution with parameter $V_{1}(z \cap \overline{\mathbf{h}}) \cdot b(u) \cdot a(\mathbf{s})$. Now, we apply this fact together with Lemma 3.4 and Proposition 3.2 to conclude that for $j=0,1$

$$
\begin{gathered}
\int \mathbf{1}\{\# T=n\} \mathbb{Q}_{(\overline{\mathbf{P}}, \boldsymbol{\beta}, \tau), t}^{(j)}(\mathrm{d}(\overline{\mathbf{p}}, \mathbf{s}, T)) \\
=\left[\varrho_{1, t}^{(j)}\right]^{-1} \int \sum_{(\mathbf{p}, \mathbf{s}, 1) \in m_{t}^{d-1}} V_{j}(\overline{\mathbf{p}}) \cdot \mathbf{1}\left\{c(\overline{\mathbf{p}}) \in[0,1]^{d}\right\} \mathbf{1}\left\{\#\left(m_{t} \sqcap \overline{\mathbf{p}}\right)=n\right\} \mathbb{P}_{M_{t}}\left(\mathrm{~d} m_{t}\right) \\
=2^{d-2}\left[\varrho_{1, t}^{(j)}\right]^{-1} \int \ldots \int \sum_{z \in y_{s-1}} V_{j}(z \cap \overline{\mathbf{h}}) \mathbf{1}\left\{c(z \cap \overline{\mathbf{h}}) \in[0,1]^{d}\right\} \\
\cdot \mathbf{1}\left\{\#\left(z \cap \overline{\mathbf{h}} \cap\left[\bigcup_{i=1}^{d-2} \partial y_{t-s_{i}}^{(i)} \cup \partial y_{t-s_{d-1}}^{+} \cup \partial y_{t-s_{d-1}}^{-}\right]\right)=n\right\} \\
\mathbb{P}_{\underline{Y}}^{\otimes d}\left(\mathrm{~d}\left(\underline{y}^{(1)}, \ldots \underline{y}^{(d-2)}, \underline{y}^{+}, \underline{y}^{-}\right)\right) \mathbb{P}_{Y_{s_{d-1}}}\left(\mathrm{~d} y_{s_{d-1}}\right) \\
\cdot \mathbf{1}\left\{0<s_{1}<\ldots<s_{d-1}<t\right\} \mathrm{d} s_{1} \ldots \mathrm{d} s_{d-1} \Lambda^{\otimes(d-1)}(\mathrm{d} \mathbf{h})
\end{gathered}
$$

and hence

$$
\begin{aligned}
\int \mathbf{1}\{\# T=n\} \mathbb{Q}_{(\overline{\mathbf{P}}, \boldsymbol{\beta}, \tau), t}^{(j)}(\mathrm{d}(\overline{\mathbf{p}}, \mathbf{s}, T)) \\
=2^{d-2}\left[\varrho_{1, t}^{(j)}\right]^{-1} \int \ldots \int \sum_{z \in y_{s_{d-1}}} V_{j}(z \cap \overline{\mathbf{h}}) \mathbf{1}\left\{c(z \cap \overline{\mathbf{h}}) \in[0,1]^{d}\right\} \\
\cdot \frac{\left[V_{1}(z \cap \overline{\mathbf{h}}) b(u) a(\mathbf{s})\right]^{n}}{n !} e^{-V_{1}(z \cap \overline{\mathbf{h}}) b(u) a(\mathbf{s})} \mathbb{P}_{Y_{s_{d-1}}}\left(\mathrm{~d} y_{s_{d-1}}\right) \\
\cdot \mathbf{1}\left\{0<s_{1}<\ldots<s_{d-1}<t\right\} \mathrm{d} s_{1} \ldots \mathrm{d} s_{d-1} \Lambda^{\otimes(d-1)}(\mathrm{d} \mathbf{h}) .
\end{aligned}
$$

For the stationary STIT tessellation $Y_{s_{d-1}}$ we consider the induced one-dimensional tessellation $Y_{s_{d-1}}^{\prime}=Y_{s_{d-1}} \cap \overline{\mathbf{h}}$ as a marked point process (centers of the segments, marked with the lengths of the segments). Its intensity, that is, the mean number of segment centres per unit length on $\overline{\mathbf{h}}$ is equal to $b(u) s_{d-1}$.

Denote by $\mathbb{Q}_{l}$ the distribution of the length of the typical segment, which is the exponential distribution with parameter $b(u) s_{d-1}$. Then the stationarity of the 
STIT tessellation and the refined Campbell theorem for marked point processes, see Schneider and Weil (2008, Theorem 3.5.3), imply for the inner integral that

$$
\begin{aligned}
I \quad & :=\int \sum_{z \in y_{s_{d-1}}} V_{j}(z \cap \overline{\mathbf{h}}) \mathbf{1}\left\{c(z \cap \overline{\mathbf{h}}) \in[0,1]^{d}\right\} \\
& \cdot \frac{\left[V_{1}(z \cap \overline{\mathbf{h}}) b(u) a(\mathbf{s})\right]^{n}}{n !} e^{-V_{1}(z \cap \overline{\mathbf{h}}) b(u) a(\mathbf{s})} \mathbb{P}_{Y_{s_{d-1}}}\left(\mathrm{~d} y_{s_{d-1}}\right) \\
& =\int_{z^{\prime} \in y_{s_{d-1}}^{\prime}} V_{j}\left(z^{\prime}\right) \mathbf{1}\left\{c\left(z^{\prime}\right) \in[0,1]^{d}\right\} \frac{\left[V_{1}\left(z^{\prime}\right) b(u) a(\mathbf{s})\right]^{n}}{n !} e^{-V_{1}\left(z^{\prime}\right) b(u) a(\mathbf{s})} \mathbb{P}_{Y_{s_{d-1}}^{\prime}}\left(\mathrm{d} y_{s_{d-1}}^{\prime}\right) \\
& =b(u) s_{d-1} V_{1}\left([0,1]^{d} \cap \overline{\mathbf{h}}\right) \int x^{j} \frac{[x b(u) a(\mathbf{s})]^{n}}{n !} e^{-x b(u) a(\mathbf{s})} \mathbb{Q}_{l}(\mathrm{~d} x) \\
& =b(u) s_{d-1} V_{1}\left([0,1]^{d} \cap \overline{\mathbf{h}}\right) \int_{0}^{\infty} x^{j} \frac{[x b(u) a(\mathbf{s})]^{n}}{n !} e^{-x b(u) a(\mathbf{s})} b(u) s_{d-1} e^{-b(u) s_{d-1} x} \mathrm{~d} x .
\end{aligned}
$$

Integration yields

$$
I= \begin{cases}V_{1}\left([0,1]^{d} \cap \overline{\mathbf{h}}\right) \frac{(n+1) a(\mathbf{s})^{n} s_{d-1}^{2}}{\left(a(\mathbf{s})+s_{d-1}\right)^{n+2}} & \text { if } j=1, \\ b(u) V_{1}\left([0,1]^{d} \cap \overline{\mathbf{h}}\right) \frac{a(\mathbf{s})^{n} s_{d-1}^{2}}{\left(a(\mathbf{s})+s_{d-1}\right)^{n+1}} & \text { if } j=0 .\end{cases}
$$

Now we compute the inner integral on the right-hand side of (3.12) for the special choices $B=[0,1]^{d}$ and $k=1$ in the same way:

$$
\begin{aligned}
I_{\rho} & :=\int \sum_{z \in y_{s_{d-1}}} V_{j}(z \cap \overline{\mathbf{h}}) \mathbf{1}\left\{c(z \cap \overline{\mathbf{h}}) \in[0,1]^{d}\right\} \mathbb{P}_{Y_{s_{d-1}}}\left(\mathrm{~d} y_{s_{d-1}}\right) \\
& =\int \sum_{z^{\prime} \in y_{s_{d-1}^{\prime}}^{\prime}} V_{j}\left(z^{\prime}\right) \mathbf{1}\left\{c\left(z^{\prime}\right) \in[0,1]^{d}\right\} \mathbb{P}_{Y_{s_{d-1}}^{\prime}}\left(\mathrm{d} y_{s_{d-1}}^{\prime}\right) \\
& =b(u) s_{d-1} V_{1}\left([0,1]^{d} \cap \overline{\mathbf{h}}\right) \int x^{j} \mathbb{Q}_{l}(\mathrm{~d} x) \\
& =b(u) s_{d-1} V_{1}\left([0,1]^{d} \cap \overline{\mathbf{h}}\right) \int_{0}^{\infty} x^{j} b(u) s_{d-1} e^{-b(u) s_{d-1} x} \mathrm{~d} x
\end{aligned}
$$

and thus we obtain

$$
I_{\rho}= \begin{cases}V_{1}\left([0,1]^{d} \cap \overline{\mathbf{h}}\right) & \text { if } j=1, \\ b(u) V_{1}\left([0,1]^{d} \cap \overline{\mathbf{h}}\right) s_{d-1} & \text { if } j=0 .\end{cases}
$$

Combining these results leads to

$$
\varrho_{1, t}^{(j)}= \begin{cases}\frac{2^{d-2} t^{d-1}}{(d-1) !} \int V_{1}\left([0,1]^{d} \cap \overline{\mathbf{h}}\right) \Lambda^{\otimes(d-1)}(\mathrm{d} \mathbf{h}) & \text { if } j=1, \\ \frac{2^{d-2} t^{d}}{d(d-2) !} \int b(u) V_{1}\left([0,1]^{d}\right) \Lambda^{\otimes(d-1)}(\mathrm{dh}) & \text { if } j=0 .\end{cases}
$$

Plugging the inner integral $I$ and the expression for $\varrho_{1, t}^{(j)}$ into (4.3), yields 


$$
\begin{aligned}
& \mathrm{p}_{1,0}(n) \\
= & d(d-2) ! \int_{0}^{t} \int_{0}^{s_{d-1}} \cdots \int_{0}^{s_{2}} \frac{s_{d-1}^{2}}{t^{d}} \frac{\left(d \cdot t-2 s_{d-1}-s_{d-2}-\ldots-s_{1}\right)^{n}}{\left(d \cdot t-s_{d-1}-s_{d-2}-\ldots-s_{1}\right)^{n+1}} \mathrm{~d} s_{1} \ldots \mathrm{d} s_{d-1}
\end{aligned}
$$

and

$$
\begin{aligned}
& \mathrm{p}_{1,1}(n) \\
= & (n+1)(d-1) ! \int_{0}^{t} \int_{0}^{s_{d-1}} \cdots \int_{0}^{s_{2}} \frac{s_{d-1}^{2}}{t^{d-1}} \quad \frac{\left(d \cdot t-2 s_{d-1}-s_{d-2}-\ldots-s_{1}\right)^{n}}{\left(d \cdot t-s_{d-1}-s_{d-2}-\ldots-s_{1}\right)^{n+2}} \\
& \mathrm{~d} s_{1} \ldots \mathrm{d} s_{d-1} .
\end{aligned}
$$

The substitution $u_{i}:=t s_{i}, i \in\{1, \ldots, d-1\}$ shows that these probabilities are independent of the time parameter $t$.

\section{Acknowledgements}

The authors are indebted to the referees and the Associate Editor for their helpful hints and comments.

\section{References}

Y. Baryshnikov and J. E. Yukich. Gaussian limits for random measures in geometric probability. Ann. Appl. Probab. 15 (1A), 213-253 (2005). MR2115042.

C. Bordenave, Y. Gousseau and F. Roueff. The dead leaves model: a general tessellation modeling occlusion. Adv. in Appl. Probab. 38 (1), 31-46 (2006). MR2213962.

R. Cowan and A. K. L. Tsang. The falling-leaves mosaic and its equilibrium properties. Adv. in Appl. Probab. 26 (1), 54-62 (1994). MR1260302.

C. Deuß and C. Thäle. The mixing property of STIT tessellations revisited. NorthWest. Eur. J. Math. 2, 1-15 (2016). MR3451006.

B. Galerne and Y. Gousseau. The transparent dead leaves model. Adv. in Appl. Probab. 44 (1), 1-20 (2012). MR2951544.

H.-O. Georgii, T. Schreiber and C. Thäle. Branching random tessellations with interaction: a thermodynamic view. Ann. Probab. 43 (4), 1892-1943 (2015). MR3353818.

E. Jacob and P. Mörters. Spatial preferential attachment networks: power laws and clustering coefficients. Ann. Appl. Probab. 25 (2), 632-662 (2015). MR3313751.

J. Jordan. Degree sequences of geometric preferential attachment graphs. Adv. in Appl. Probab. 42 (2), 319-330 (2010). MR2675104.

J. Jordan and A. R. Wade. Phase transitions for random geometric preferential attachment graphs. Adv. in Appl. Probab. 47 (2), 565-588 (2015). MR3360390.

O. Kallenberg. Foundations of Modern Probability. Probability and its Applications (New York). Springer-Verlag, New York, second edition (2002). ISBN 0-38795313-2. MR1876169.

R. Lachièze-Rey. Mixing properties for STIT tessellations. Adv. in Appl. Probab. 43 (1), 40-48 (2011). MR2761143.

G. Last and M. D. Penrose. Lectures on the Poisson Process (2017+). To appear as IMS Textbook by Cambridge University Press.

S. Martínez. STIT tessellations are Bernoulli and standard. Ergodic Theory Dynam. Systems 34 (3), 876-892 (2014). MR3199798. 
S. Martínez and W. Nagel. Ergodic description of STIT tessellations. Stochastics 84 (1), 113-134 (2012). MR2876475.

S. Martínez and W. Nagel. STIT process and trees. Markov Process. Related Fields 21 (4), 847-868 (2015). MR3496228.

S. Martínez and W. Nagel. The $\beta$-mixing rate of STIT tessellations. Stochastics 88 (3), 396-414 (2016). MR3459730.

J. Mecke and L. Muche. The Poisson Voronoi tessellation. I. A basic identity. Math. Nachr. 176, 199-208 (1995). MR1361135.

J. Mecke, W. Nagel and V. Weiß. A global construction of homogeneous random planar tessellations that are stable under iteration. Stochastics 80 (1), 51-67 (2008a). MR2384821.

J. Mecke, W. Nagel and V. Weiß. The iteration of random tessellations and a construction of a homogeneous process of cell divisions. Adv. in Appl. Probab. 40 (1), 49-59 (2008b). MR2411814.

J. Mecke, W. Nagel and V. Weiß. Some distributions for I-segments of planar random homogeneous STIT tessellations. Math. Nachr. 284 (11-12), 1483-1495 (2011). MR2832660.

L. Muche. The Poisson-Voronor tessellation. II. Edge length distribution functions. Math. Nachr. 178, 271-283 (1996). MR1380713.

L. Muche. The Poisson Voronoi tessellation. III. Miles' formula. Math. Nachr. 191, 247-267 (1998). MR1621322.

W. Nagel and E. Biehler. Consistency of constructions for cell division processes. Adv. in Appl. Probab. 47 (3), 640-651 (2015). MR3406601.

W. Nagel and V. Weiß. Limits of sequences of stationary planar tessellations. Adv. in Appl. Probab. 35 (1), 123-138 (2003). MR1975507.

W. Nagel and V. Weiß. Crack STIT tessellations: characterization of stationary random tessellations stable with respect to iteration. Adv. in Appl. Probab. 37 (4), 859-883 (2005). MR2193987.

W. Nagel and V. Weiß. Mean values for homogeneous STIT tessellations in 3D. Image Anal. Stereol. 27, 29-37 (2008). DOI: 10.5566/ias.v27.p29-37.

M. D. Penrose and A. R. Wade. Limit theory for the random on-line nearestneighbor graph. Random Structures Algorithms 32 (2), 125-156 (2008). MR2387554.

M. D. Penrose and J. E. Yukich. Limit theory for random sequential packing and deposition. Ann. Appl. Probab. 12 (1), 272-301 (2002). MR1890065.

R. Schneider and W. Weil. Stochastic and Integral Geometry. Probability and its Applications (New York). Springer-Verlag, Berlin (2008). ISBN 978-3-540-788584. MR2455326.

T. Schreiber. Random dynamics and thermodynamic limits for polygonal Markov fields in the plane. Adv. in Appl. Probab. 37 (4), $884-907$ (2005). MR2193988.

T. Schreiber. Non-homogeneous polygonal Markov fields in the plane: graphical representations and geometry of higher order correlations. J. Stat. Phys. 132 (4), 669-705 (2008). MR2429704.

T. Schreiber. Polygonal web representation for higher order correlation functions of consistent polygonal Markov fields in the plane. J. Stat. Phys. 140 (4), 752-783 (2010). MR2670740.

T. Schreiber, M. D. Penrose and J. E. Yukich. Gaussian limits for multidimensional random sequential packing at saturation. Comm. Math. Phys. 272 (1), 167-183 
(2007). MR2291806.

T. Schreiber and C. Thäle. Second-order properties and central limit theory for the vertex process of iteration infinitely divisible and iteration stable random tessellations in the plane. Adv. in Appl. Probab. 42 (4), 913-935 (2010). MR2796670.

T. Schreiber and C. Thäle. Intrinsic volumes of the maximal polytope process in higher dimensional STIT tessellations. Stochastic Process. Appl. 121 (5), 9891012 (2011). MR2775104.

T. Schreiber and C. Thäle. Second-order theory for iteration stable tessellations. Probab. Math. Statist. 32 (2), 281-300 (2012). MR3021459.

T. Schreiber and C. Thäle. Geometry of iteration stable tessellations: connection with Poisson hyperplanes. Bernoulli 19 (5A), 1637-1654 (2013a). MR3129028.

T. Schreiber and C. Thäle. Limit theorems for iteration stable tessellations. Ann. Probab. 41 (3B), 2261-2278 (2013b). MR3098072.

C. Thäle. The distribution of the number of nodes in the relative interior of the typical I-segment in homogeneous planar anisotropic STIT tessellations. Comment. Math. Univ. Carolin. 51 (3), 503-512 (2010). MR2741883.

C. Thäle and V. Weiß. New mean values for homogeneous spatial tessellations that are stable under iteration. Image Anal. Stereol. 29 (3), 143-157 (2010). MR2780338.

C. Thäle and V. Weiß. The combinatorial structure of spatial STIT tessellations. Discrete Comput. Geom. 50 (3), 649-672 (2013). MR3102583.

C. Thäle, V. Weiß and W. Nagel. Spatial STIT tessellations: distributional results for I-segments. Adv. in Appl. Probab. 44 (3), 635-654 (2012). MR3024603.

A. R. Wade. Asymptotic theory for the multidimensional random on-line nearest-neighbour graph. Stochastic Process. Appl. 119 (6), 1889-1911 (2009). MR2519349. 